\title{
PHOTOMETRY OF THE RS CVN TYPE ECLIPSING BINARY UV PISCIUM*
}

\author{
P. VIVEKANANDA RAO and M. B. K. SARMA \\ Centre of Advanced Study in Astronomy, Osmania University, Hyderabad, India
}

(Received 12 August, 1983)

\begin{abstract}
The analysis of the UBV photoelectric study of the short period RS CVn eclipsing binary, UV Psc, has suggested that the primary is a transit with $\theta_{e}=27^{\circ}, i=88.5$, and $k=0.75$. The spectral type and luminosity of the hotter component is estimated to be G4-6V and that of the cooler component to be $\mathrm{K} 0-2 \mathrm{~V}$. Absolute dimensions for the components of UV Psc were derived by combining the present analysis with that of the spectroscopic analysis given by Popper.

The out-of-eclipse observations have showed large amount of scatter and an investigation of this showed that hotter component could be an intrinsic variable. No periodicity for this variation has been fixed due to lack of sufficient data.
\end{abstract}

\section{Introduction}

The light variability of UV Piscium (BD $\left.+6^{\circ} 189=\mathrm{BV} 149\right)$ was noticed from the photographic patrol plates and photographic light curves for this system were published by Huth (1959) and Strohmeier and Knigge (1960). Hall (1976) classified UV Psc as a member of the short period RS CVn group. These binaries exhibit various peculiarities such as $\mathrm{H}$ and $\mathrm{K}$ of $\mathrm{Ca} I \mathrm{II}$ in emission indicating the presence of active chromospheres and coronae and also radio and soft $\mathrm{X}$-ray emission, which strongly suggest the presence of a large scale solar-type activity such as the spots on the surface of one or both the components. As a result, these binaries often show a wave like distortion (Hall, 1981; Rodono, 1981) in their out-of-eclipse light curves.

The presence of this photometric wave in the RS CVn binaries distorts the light curve such that the times of primary and secondary minima will be displaced and the shape of the light curve during the eclipses will also be distorted. It is very essential to correct the observations for the effect of this wave before one uses them for determining the times of minima or for solving orbital elements from the light curves. Before undertaking the analysis for the computation of elements, it is essential to know which component of the system (hotter or cooler) is responsible for the wave nature so that suitable corrections can be applied to the observations of the primary and secondary eclipses. Photometric analysis of UV Psc by Carr (1969), Oliver (1974), and Sadik (1979) were concentrated only on the solution of the light curves without taking into consideration the properties of the distortion

\footnotetext{
* Paper presented at the Lembang-Bamberg IAU Colloquium No. 80 on 'Double Stars: Physical Properties and Generic Relations', held at Bandung, Indonesia, 3-7 June, 1983.
} 
wave, the source responsible for it, its effect on the period determination and the nature of the intrinsic variation.

However, by combining our present observations with those of Carr (1969), Oliver (1974), and Sadik (1979), the authors (Vivekananda Rao and Sarma, 1983b; hereafter referred to as Paper II) have showed that UV Psc exhibits a doublepeaked distortion wave - suggesting the presence of two cool dark regions located on the surface of the hotter G4-6 component. By use of the constants derived for the wave, the observations during the primary minima were corrected for distortion and the times of minima were determined. With these corrected minima and other times of minima available in the literature, a period study was undertaken by the authors (Vivekananda Rao and Sarma, 1983a; hereafter referred to as Paper I). From this study it was found that no reliable period changes took place in this system during the interval 1966-81.

The present communication mainly deals with the nature and source of the intrinsic variation, the light curve solution after correcting for the distortion wave effect and the evolutionary nature of the system.

\section{Observations}

UV Psc was observed for 47 nights in $U$ passband and 54 nights in $B$ and $V$ passbands on the standard $U B V$ system during 1976-77, 1977-78, and 1978-79 observing seasons using the $1.22 \mathrm{~m}$ reflecting telescope of the Japal-Rangapur Observatory. The details of the photometric equipment and the reduction techniques were described in an earlier paper (Vivekananda Rao and Sarma, 1981b). The $U B V$ light curves obtained during these three observing seasons were already published (Vivekananda Rao and Sarma, 1981c).

\section{Rectification}

In Paper II it was shown that the hotter component is responsible for the distortion wave in UV Psc. In Section 5 of this paper it is also shown that the primary eclipse is a transit where the cooler component is transiting over the hotter component. By virtue of these properties, all the observations obtained during October 1976-December 1978 were corrected for the distortion wave effects with the aid of the equation

$$
\begin{aligned}
l^{\mathrm{cor}}= & l^{\mathrm{obs}}-\left(1-f^{\mathrm{tr}}\right)\left[A_{1}(\text { wave }) \cos \theta+B_{1} \sin \theta\right]- \\
& -\left(1-f^{\mathrm{tr}}\right)\left[A_{2}(\text { wave }) \cos 2 \theta+B_{2} \sin 2 \theta\right],
\end{aligned}
$$

where

$$
f^{\mathrm{tr}}(x, k, p)=\tau(x, k) \alpha_{0}^{\mathrm{tr}}(x, k, p) n
$$

is the fractional loss of light of the hotter and spotted star during the primary 
eclipse. A detailed derivation of $A_{1}$ and $A_{2}$ (wave-constants) for each year of observations and the procedure adopted for calculating $f$ 's during the primary minimum were given in Papers I and II, respectively. The value of $(1-f)$ is equal to unity for points outside the eclipse and also during the secondary minimum, which is the eclipse of the unspotted cool star. All the observed points corrected according to the above equation were converted into magnitudes (by taking the magnitude corresponding to unit light intensity for the respective years) to obtain a unified light curve for each passband during Oct. 1976-Dec. 1978. The observations of Carr (1969), Oliver (1974), and Sadik (1979) were not included in the unified plot in order to avoid the uncertainties involved in transforming their observations into our system because of the differences in the photometric equipment, the comparison and check stars used. The light curves obtained during our period of observation should be identical within the limits of the observational errors as we had removed the effects of the wave from the observations. Figure 1a shows the combined plot of actual observations (not corrected for the wave) whereas Figure $1 \mathrm{~b}$ shows the combined plot of observations that are corrected for the wave for the $V$-passband. From these two figures (Figures $1 \mathrm{la}$ and $1 \mathrm{~b}$ ) it is evident that the observations of all three years could be merged satisfactorily in all phases except that of the primary eclipse portion. Even here, the differences in the depths observed during the interval had reduced considerably. These conclusions

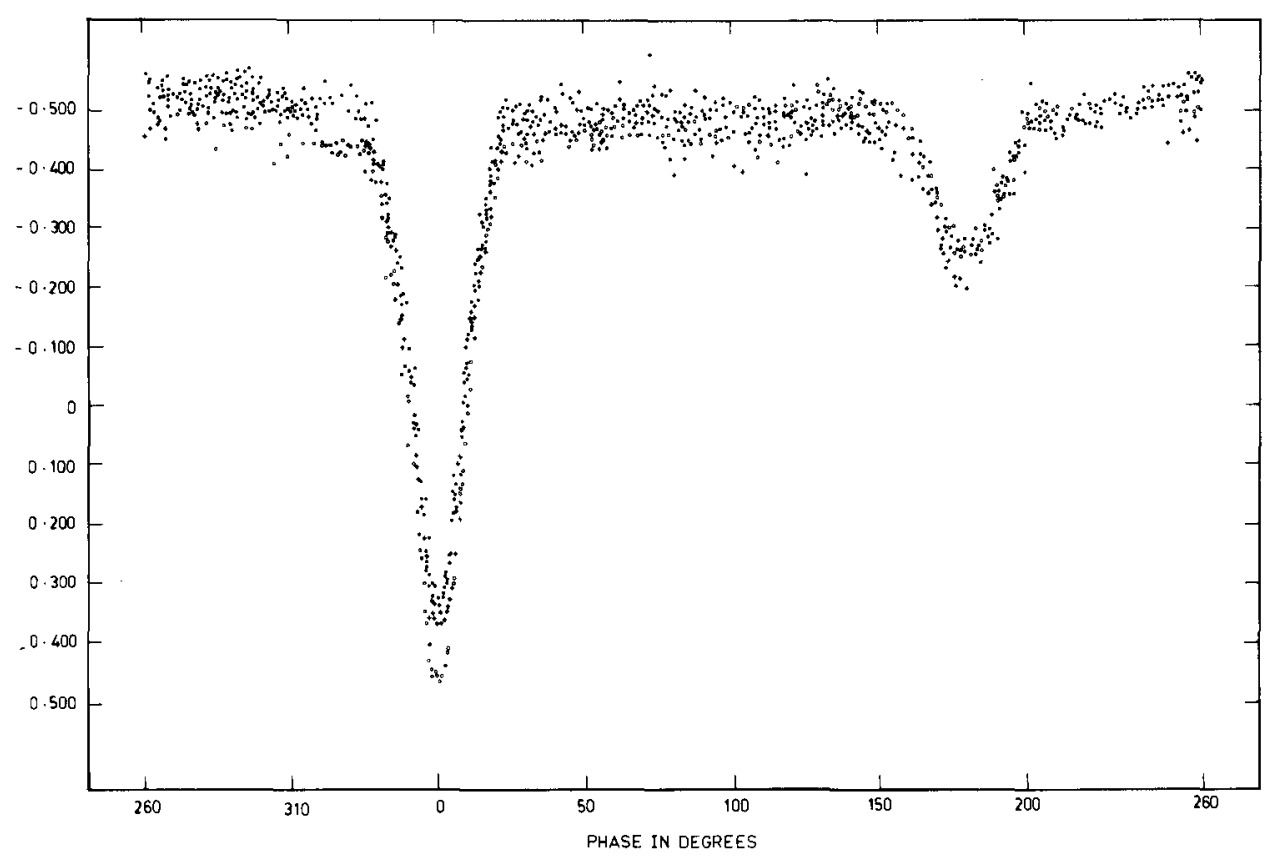

Fig. 1a. UV Psc: Plot of observed $\Delta V$ versus phase in degrees for the combined light curve. $O$ represents 1976-77 data. + represents 1977-78 data. represents 1978-79 data. The effect of the distortion wave can be seen in both primary and secondary minima. 


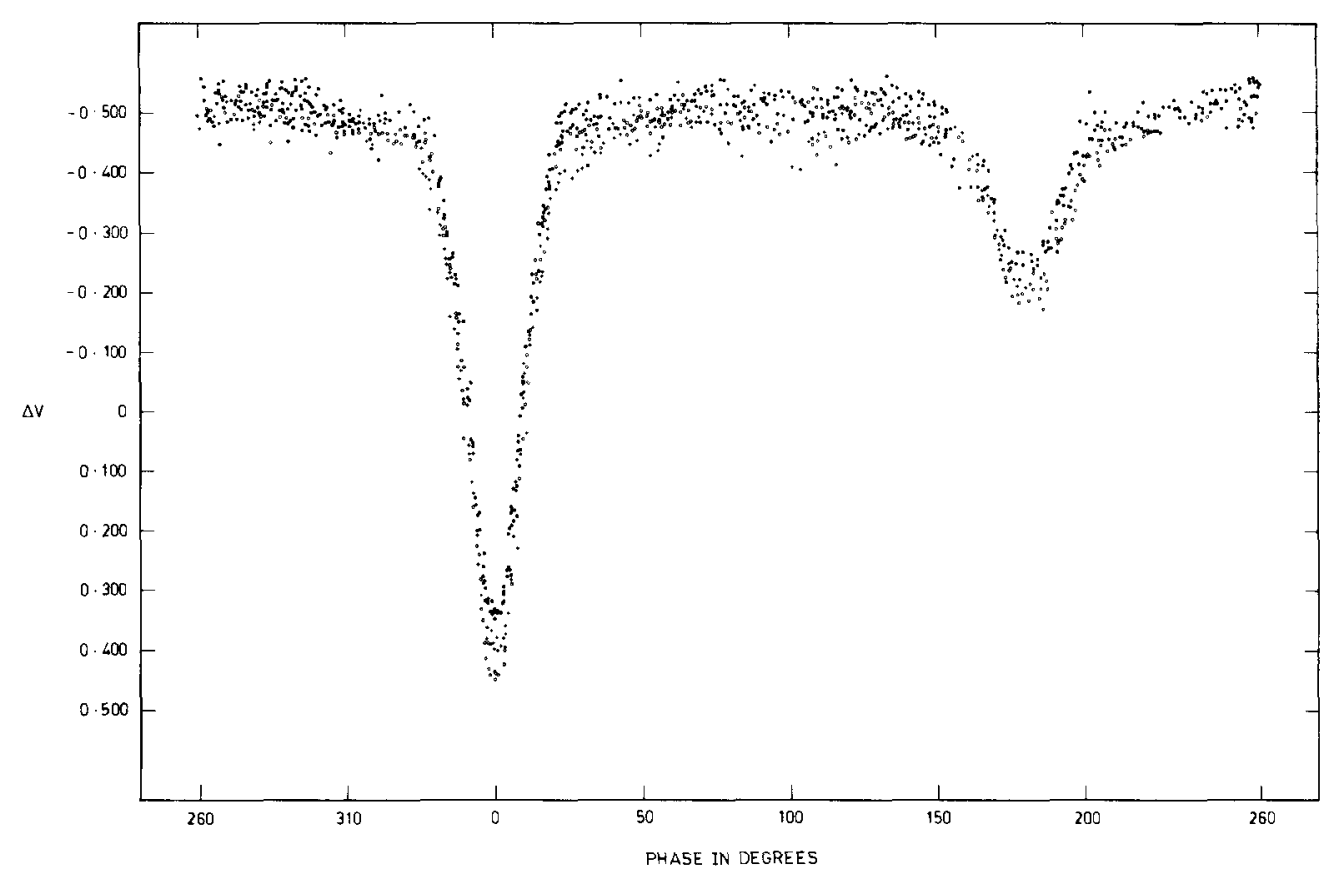

Fig. 1b. UV Psc: Plot of observed $\Delta V$ versus phase in degrees for the combined light curve after the removal of the distortion wave. Symbols same as in Figure la. It can be seen that except at the zero phase the points at other phases have merged satisfactorily.

hold good for the combined light curves of $B$ and $U$ passbands also. Assuming that the differences at the primary minimum are of intrinsic nature, we have used the averaged light curves (corrected for the wave effects) in our further analysis.

Treating the wave effects removed unified light curves in $U B V$ passbands as the real observed ones, these were then normalised to unit light intensity at maximum by adding $-0^{m} \cdot 495,-0^{m} \cdot 258$, and $+0^{m} \cdot 011$ in $V, B$, and $U$ passbands, respectively. These values are the average magnitudes at phase angles $90^{\circ}$ and $270^{\circ}$ of the respective unified light curves. The observations were then grouped together to form normal points in all passbands and are given in Tables Ia, Ib, and Ic.

A preliminary (graphical) study of our light curves has yielded a value of $27^{\circ}$ for the angle of external tangency, $\theta_{e}$, which agrees well with the one obtained $\left(\theta_{e}=26^{\circ} 5\right)$ by Carr (1969) and Sadik (1979). Using this limit, the light outside the eclipses i.e., light from $27^{\circ}<\theta<153^{\circ}$ and $270^{\circ}<\theta<333^{\circ}$ was represented by the Fourier expression:

$$
l=\sum_{n=0}^{4} A_{n} \cos n \theta+\sum_{n=1}^{4} B_{n} \sin n \theta
$$

The values of $A_{n}$ and $B_{n}$ along with their probable errors were determined by the 
TABLE Ia

UV Psc: normal points in yellow

\begin{tabular}{|c|c|c|c|c|c|}
\hline $\begin{array}{l}\text { Phase } \\
\text { (in degrees) }\end{array}$ & $\begin{array}{l}\Delta V \\
(\operatorname{Var}-\operatorname{Comp})\end{array}$ & $\begin{array}{l}\text { No. of } \\
\text { points }\end{array}$ & $\begin{array}{l}\text { Phase } \\
\text { (in degrees) }\end{array}$ & $\begin{array}{l}\Delta V \\
\text { (Var-Comp) }\end{array}$ & $\begin{array}{l}\text { No. of } \\
\text { points }\end{array}$ \\
\hline 0.010 & +0.379 & 5 & 55.668 & -0.485 & 12 \\
\hline 0.742 & +0.388 & 5 & 58.724 & -0.492 & 9 \\
\hline 1.450 & +0.368 & 4 & 61.149 & -0.514 & 8 \\
\hline 2.587 & +0.334 & 4 & 64.669 & -0.504 & 10 \\
\hline 3.276 & +0.357 & 4 & 68.665 & -0.509 & 11 \\
\hline 3.650 & +0.378 & 4 & 71.572 & -0.521 & 10 \\
\hline 4.334 & +0.266 & 4 & 74.851 & -0.515 & 11 \\
\hline 5.367 & +0.280 & 4 & 78.699 & -0.498 & 8 \\
\hline 5.764 & +0.221 & 5 & 81.694 & -0.489 & 8 \\
\hline 6.390 & +0.171 & 5 & 84.353 & -0.491 & 10 \\
\hline 7.295 & +0.144 & 3 & 87.335 & -0.508 & 8 \\
\hline 7.748 & +0.122 & 4 & 89.882 & -0.491 & 4 \\
\hline 8.012 & +0.117 & 4 & 92.275 & -0.505 & 7 \\
\hline 8.557 & +0.065 & 5 & 95.561 & -0.487 & 7 \\
\hline 9.329 & +0.016 & 4 & 98.427 & -0.504 & 5 \\
\hline 9.883 & -0.044 & 5 & 101.163 & -0.495 & 5 \\
\hline 10.511 & -0.029 & 5 & 104.790 & -0.489 & 7 \\
\hline 11.496 & -0.090 & 5 & 107.606 & -0.492 & 9 \\
\hline 12.096 & -0.126 & 5 & 110.270 & -0.493 & 10 \\
\hline 12.740 & -0.150 & 5 & 113.353 & -0.486 & 8 \\
\hline 13.785 & -0.224 & 5 & 116.507 & -0.497 & 11 \\
\hline 14.502 & -0.219 & 4 & 119.739 & -0.488 & 9 \\
\hline 15.122 & -0.251 & 5 & 122.602 & -0.509 & 9 \\
\hline 15.628 & -0.250 & 4 & 125.844 & -0.492 & 9 \\
\hline 16.573 & -0.288 & 4 & 128.447 & -0.498 & 9 \\
\hline 17.104 & -0.329 & 4 & 131.461 & -0.511 & 9 \\
\hline 17.669 & -0.334 & 4 & 134.659 & -0.505 & 10 \\
\hline 18.222 & -0.336 & 4 & 137.529 & -0.496 & 8 \\
\hline 18.682 & -0.379 & 4 & 140.446 & -0.494 & 11 \\
\hline 19.264 & -0.378 & 4 & 143.755 & -0.496 & 9 \\
\hline 20.551 & -0.404 & 4 & 146.784 & -0.475 & 9 \\
\hline 21.275 & -0.427 & 4 & 149.619 & -0.472 & 9 \\
\hline 21,808 & -0.430 & 4 & 152.084 & -0.474 & 4 \\
\hline 22.418 & -0.446 & 4 & 153.398 & -0.493 & 3 \\
\hline 23.120 & -0.472 & 5 & 154.967 & -0.452 & 3 \\
\hline 24.395 & -0.472 & 4 & 156.558 & -0.451 & 3 \\
\hline 25.796 & -0.479 & 3 & 158.884 & -0.429 & 3 \\
\hline 26.747 & -0.458 & 3 & 161.684 & -0.406 & 4 \\
\hline 27.707 & -0.490 & 2 & 163.985 & -0.397 & 3 \\
\hline 28.936 & -0.458 & 7 & 164.664 & -0.398 & 3 \\
\hline 31.574 & -0.469 & 4 & 165.689 & -0.375 & 3 \\
\hline 34.653 & -0.461 & 9 & 166.296 & -0.379 & 4 \\
\hline 36.742 & -0.493 & 7 & 167.922 & -0.363 & 4 \\
\hline 40.522 & -0.481 & 7 & 169.500 & -0.326 & 4 \\
\hline 43.377 & -0.498 & 5 & 170.265 & -0.324 & 4 \\
\hline 46.337 & -0.483 & 8 & 171.557 & -0.272 & 4 \\
\hline 49.259 & -0.492 & 9 & 172.217 & -0.273 & 4 \\
\hline 52.657 & -0.481 & 9 & 173.337 & -0.240 & 3 \\
\hline
\end{tabular}


Table Ia (continued)

\begin{tabular}{|c|c|c|c|c|c|}
\hline $\begin{array}{l}\text { Phase } \\
\text { (in degrees) }\end{array}$ & $\begin{array}{l}\Delta V \\
\text { (Var-Comp) }\end{array}$ & $\begin{array}{l}\text { No. of } \\
\text { points }\end{array}$ & $\begin{array}{l}\text { Phase } \\
\text { (in degrees) }\end{array}$ & $\begin{array}{l}\Delta V \\
\text { (Var-Comp) }\end{array}$ & $\begin{array}{l}\text { No. of } \\
\text { points }\end{array}$ \\
\hline 174.517 & -0.253 & 3 & 278.648 & -0.505 & 9 \\
\hline 175.800 & -0.226 & 4 & 281.714 & -0.512 & 13 \\
\hline 177.341 & -0.242 & 3 & 284.511 & -0.510 & 9 \\
\hline 178.279 & -0.217 & 4 & 287.519 & -0.517 & 11 \\
\hline 180.002 & -0.210 & 4 & 290.566 & -0.502 & 12 \\
\hline 181.900 & -0.246 & 3 & 293.569 & -0.522 & 9 \\
\hline 182.673 & -0.226 & 3 & 296.566 & -0.502 & 14 \\
\hline 184.234 & -0.251 & 3 & 299.688 & -0.502 & 9 \\
\hline 185.304 & -0.208 & 3 & 302.837 & -0.495 & 9 \\
\hline 186.303 & -0.242 & 4 & 306.292 & -0.481 & 10 \\
\hline 187.438 & -0.244 & 4 & 309.408 & -0.484 & 10 \\
\hline 188.518 & -0.284 & 3 & 312.175 & -0.480 & 9 \\
\hline 190.167 & -0.302 & 3 & 315.342 & -0.483 & 7 \\
\hline 191.053 & -0.308 & 4 & 318.516 & -0.462 & 7 \\
\hline 191.576 & -0.328 & 3 & 321.350 & -0.477 & 9 \\
\hline 192.655 & -0.329 & 4 & 324.093 & -0.477 & 5 \\
\hline 193.454 & -0.350 & 4 & 327.760 & -0.464 & 6 \\
\hline 194.930 & -0.368 & 4 & 330.667 & -0.479 & 4 \\
\hline 196.020 & -0.355 & 4 & 333.178 & -0.474 & 3 \\
\hline 197.407 & -0.403 & 4 & 334.181 & -0.461 & 4 \\
\hline 198.424 & -0.425 & 3 & 335.306 & -0.429 & 4 \\
\hline 199.681 & -0.395 & 4 & 336.675 & -0.438 & 5 \\
\hline 200.714 & -0.440 & 4 & 337.623 & -0.402 & 5 \\
\hline 202.213 & -0.466 & 4 & 338.640 & -0.427 & 5 \\
\hline 204.298 & -0.467 & 3 & 339.950 & -0.405 & 4 \\
\hline 204.980 & -0.440 & 4 & 341.455 & -0.379 & 3 \\
\hline 206.104 & -0.465 & 4 & 341.731 & -0.351 & 4 \\
\hline 207.725 & -0.466 & 4 & 342.664 & -0.282 & 4 \\
\hline 210.325 & -0.458 & 6 & 343.301 & -0.331 & 5 \\
\hline 213.575 & -0.460 & 6 & 343.524 & -0.290 & 4 \\
\hline 216.468 & -0.472 & 3 & 344.502 & -0.234 & 3 \\
\hline 218.839 & -0.482 & 3 & 345.311 & -0.226 & 4 \\
\hline 221.435 & -0.478 & 7 & 345.799 & -0.236 & 3 \\
\hline 224.464 & -0.485 & 6 & 346.498 & -0.210 & 4 \\
\hline 227.328 & -0.495 & 3 & 347.536 & -0.175 & 4 \\
\hline 231.618 & -0.505 & 5 & 347.973 & -0.115 & 5 \\
\hline 235.443 & -0.496 & 4 & 348.792 & -0.094 & 4 \\
\hline 238.502 & -0.501 & 4 & 349.832 & -0.070 & 4 \\
\hline 241.440 & -0.508 & 7 & 350.468 & -0.004 & 4 \\
\hline 244.654 & -0.521 & 4 & 351.633 & +0.004 & 5 \\
\hline 247.913 & -0.514 & 5 & 352.257 & +0.029 & 3 \\
\hline 251.806 & -0.525 & 4 & 352.804 & +0.095 & 3 \\
\hline 254.471 & -0.513 & 9 & 353.396 & +0.152 & 5 \\
\hline 257.553 & -0.516 & 9 & 354.459 & +0.211 & 4 \\
\hline 260.169 & -0.524 & 9 & 354.844 & +0.210 & 3 \\
\hline 263.424 & -0.501 & 10 & 355.655 & +0.318 & 4 \\
\hline 266.703 & -0.510 & 9 & 356.204 & +0.277 & 4 \\
\hline 268.652 & -0.520 & 4 & 356.754 & +0.310 & 4 \\
\hline 269.963 & -0.500 & 3 & 357.564 & +0.348 & 3 \\
\hline 272.779 & -0.515 & 8 & 358.197 & +0.400 & 5 \\
\hline 275.693 & -0.518 & 12 & 358.831 & +0.360 & 5 \\
\hline
\end{tabular}


TABLE Ib

UV Psc: normal points in blue

\begin{tabular}{|c|c|c|c|c|c|}
\hline $\begin{array}{l}\text { Phase } \\
\text { (in degrees) }\end{array}$ & $\begin{array}{l}\Delta B \\
\text { (Var-Comp) }\end{array}$ & $\begin{array}{l}\text { No. of } \\
\text { points }\end{array}$ & $\begin{array}{l}\text { Phase } \\
\text { (in degrees) }\end{array}$ & $\begin{array}{l}\Delta B \\
\text { (Var-Comp) }\end{array}$ & $\begin{array}{l}\text { No. of } \\
\text { points }\end{array}$ \\
\hline 0.778 & +0.735 & 4 & 56.831 & -0.214 & 11 \\
\hline 1.388 & +0.740 & 4 & 60.471 & -0.235 & 10 \\
\hline 2.585 & +0.721 & 4 & 63.382 & -0.228 & 9 \\
\hline 3.072 & +0.720 & 4 & 66.408 & -0.239 & 7 \\
\hline 3.648 & +0.682 & 4 & 69.708 & -0.242 & 11 \\
\hline 4.445 & +0.644 & 4 & 71.851 & -0.237 & 8 \\
\hline 4.834 & +0.587 & 3 & 75.322 & -0.255 & 9 \\
\hline 5.795 & +0.585 & 5 & 78.641 & -0.251 & 6 \\
\hline 6.027 & +0.583 & 5 & 81.147 & -0.232 & 9 \\
\hline 6.416 & +0.507 & 5 & 84.306 & -0.233 & 12 \\
\hline 7.064 & +0.494 & 4 & 87.795 & -0.244 & 5 \\
\hline 7.901 & +0.441 & 4 & 90.099 & -0.252 & 7 \\
\hline 8.569 & +0.357 & 5 & 92.708 & -0.246 & 5 \\
\hline 9.074 & +0.352 & 4 & 96.469 & -0.242 & 9 \\
\hline 9.690 & +0.305 & 4 & 99.080 & -0.231 & 4 \\
\hline 10.294 & +0.233 & 4 & 102.386 & -0.240 & 6 \\
\hline 11.128 & +0.254 & 5 & 105.606 & -0.239 & 6 \\
\hline 11.564 & +0.176 & 4 & 108.454 & -0.214 & 12 \\
\hline 11.922 & +0.200 & 4 & 111.910 & -0.221 & 12 \\
\hline 12.548 & +0.150 & 4 & 115.781 & -0.228 & 10 \\
\hline 13.400 & +0.088 & 4 & 118.981 & -0.222 & 10 \\
\hline 14.259 & +0.047 & 4 & 122.530 & -0.234 & 10 \\
\hline 14.728 & +0.042 & 5 & 125.662 & -0.214 & 9 \\
\hline 15.676 & 0.000 & 4 & 128.196 & -0.239 & 9 \\
\hline 16.174 & +0.012 & 5 & 131.818 & -0.254 & 8 \\
\hline 16.708 & -0.034 & 4 & 134.802 & -0.234 & 11 \\
\hline 17.545 & -0.072 & 4 & 138.309 & -0.230 & 9 \\
\hline 18.021 & -0.073 & 5 & 140.913 & -0.218 & 9 \\
\hline 18.704 & -0.095 & 4 & 144.479 & -0.212 & 11 \\
\hline 19.530 & -0.131 & 5 & 147.379 & -0.206 & 9 \\
\hline 20.306 & -0.132 & 4 & 149.827 & -0.212 & 5 \\
\hline 21.566 & -0.127 & 4 & 151.949 & -0.220 & 7 \\
\hline 22.128 & -0.189 & 5 & 154.014 & -0.234 & 3 \\
\hline 22.808 & -0.181 & 4 & 155.691 & -0.202 & 3 \\
\hline 23.429 & -0.203 & 5 & 157.186 & -0.165 & 3 \\
\hline 24.830 & -0.216 & 4 & 160.166 & -0.174 & 4 \\
\hline 26.301 & -0.221 & 3 & 162.728 & -0.186 & 4 \\
\hline 27.611 & -0.203 & 3 & 164.750 & -0.135 & 3 \\
\hline 28.534 & -0.200 & 4 & 165.738 & -0.120 & 3 \\
\hline 30.214 & -0.199 & 11 & 166.377 & -0.102 & 3 \\
\hline 33.183 & -0.215 & 11 & 167.321 & -0.091 & 4 \\
\hline 36.262 & -0.210 & 10 & 169.107 & -0.072 & 3 \\
\hline 38.738 & -0.211 & 3 & 170.372 & -0.053 & 4 \\
\hline 41.640 & -0.231 & 7 & 171.035 & -0.054 & 4 \\
\hline 44.631 & -0.229 & 5 & 172.028 & -0.042 & 4 \\
\hline 47.632 & -0.221 & 12 & 173.590 & -0.020 & 4 \\
\hline 51.832 & -0.218 & 10 & 174.847 & -0.017 & 4 \\
\hline 54.314 & -0.201 & 6 & 175.755 & +0.002 & 4 \\
\hline
\end{tabular}


Table Ib (continued)

\begin{tabular}{|c|c|c|c|c|c|}
\hline $\begin{array}{l}\text { Phase } \\
\text { (in degrees) }\end{array}$ & $\begin{array}{l}\Delta V \\
\text { (Var-Comp) }\end{array}$ & $\begin{array}{l}\text { No. of } \\
\text { points }\end{array}$ & $\begin{array}{l}\text { Phase } \\
\text { (in degrees) }\end{array}$ & $\begin{array}{l}\Delta V \\
\text { (Var-Comp) }\end{array}$ & $\begin{array}{l}\text { No. of } \\
\text { points }\end{array}$ \\
\hline 176.860 & +0.026 & 4 & 285.205 & -0.246 & 8 \\
\hline 178.443 & +0.015 & 4 & 288.230 & -0.239 & 12 \\
\hline 179.972 & +0.024 & 4 & 291.520 & -0.252 & 12 \\
\hline 181.872 & -0.016 & 4 & 294.836 & -0.244 & 10 \\
\hline 183.338 & -0.007 & 3 & 297.282 & -0.244 & 12 \\
\hline 184.819 & -0.016 & 3 & 300.632 & -0.221 & 10 \\
\hline 185.760 & -0.001 & 3 & 303.596 & -0.227 & 6 \\
\hline 186.772 & -0.019 & 3 & 306.753 & -0.216 & 11 \\
\hline 187.594 & -0.016 & 4 & 309.704 & -0.214 & 9 \\
\hline 188.754 & -0.048 & 4 & 312.627 & -0.198 & 8 \\
\hline 190.276 & -0.063 & 3 & 315.260 & -0.209 & 7 \\
\hline 191.788 & -0.068 & 4 & 318.431 & -0.212 & 7 \\
\hline 192.461 & -0.082 & 4 & 321.288 & -0.200 & 10 \\
\hline 193.237 & -0.104 & 4 & 325.637 & -0.202 & 8 \\
\hline 194.512 & -0.098 & 3 & 329.132 & -0.206 & 2 \\
\hline 195.506 & -0.115 & 3 & 330.583 & -0.197 & 3 \\
\hline 196.463 & -0.135 & 4 & 332.314 & -0.238 & 2 \\
\hline 197.478 & -0.136 & 4 & 333.990 & -0.197 & 4 \\
\hline 198.801 & -0.148 & 4 & 335.108 & -0.170 & 5 \\
\hline 200.425 & -0.171 & 4 & 336.354 & -0.182 & 5 \\
\hline 201.624 & -0.177 & 4 & 337.593 & -0.163 & 5 \\
\hline 202.802 & -0.171 & 3 & 338.529 & -0.127 & 5 \\
\hline 204.176 & -0.186 & 4 & 339.963 & -0.139 & 5 \\
\hline 205.335 & -0.195 & 3 & 341.392 & -0.112 & 4 \\
\hline 206.184 & -0.197 & 3 & 342.003 & -0.051 & 5 \\
\hline 208.135 & -0.187 & 5 & 342.803 & -0.029 & 5 \\
\hline 211.207 & -0.177 & 7 & 343.731 & -0.018 & 5 \\
\hline 213.980 & -0.199 & 4 & 344.169 & -0.002 & 4 \\
\hline 217.327 & -0.204 & 5 & 345.200 & +0.021 & 5 \\
\hline 221.366 & -0.198 & 8 & 346.464 & +0.090 & 5 \\
\hline 224.745 & -0.221 & 7 & 347.423 & +0.140 & 5 \\
\hline 229.454 & -0.252 & 4 & 348.137 & +0.138 & 5 \\
\hline 233.974 & -0.247 & 7 & 348.634 & +0.161 & 5 \\
\hline 238.462 & -0.223 & 6 & 349.507 & +0.185 & 3 \\
\hline 242.196 & -0.232 & 7 & 350.344 & +0.265 & 4 \\
\hline 245.667 & -0.234 & 5 & 351.365 & +0.318 & 3 \\
\hline 248.379 & -0.224 & 3 & 352.447 & +0.418 & 4 \\
\hline 252.556 & -0.262 & 7 & 352.870 & +0.414 & 4 \\
\hline 255.050 & -0.222 & 9 & 353.750 & +0.485 & 5 \\
\hline 258.872 & -0.251 & 13 & 354.256 & +0.547 & 4 \\
\hline 262.577 & -0.261 & 6 & 354.978 & +0.593 & 5 \\
\hline 264.216 & -0.255 & 9 & 355.837 & +0.656 & 4 \\
\hline 267.602 & -0.255 & 11 & 356.659 & +0.691 & 5 \\
\hline 270.099 & -0.267 & 5 & 357.244 & +0.684 & 4 \\
\hline 273.761 & -0.267 & 11 & 357.924 & +0.698 & 4 \\
\hline 276.378 & -0.255 & 8 & 358.426 & +0.714 & 4 \\
\hline 279.118 & -0.245 & 8 & 359.165 & +0.724 & 4 \\
\hline 282.559 & -0.257 & 16 & 359.984 & +0.754 & 4 \\
\hline
\end{tabular}


TABLE Ic

UV Psc: normal points in ultraviolet

\begin{tabular}{|c|c|c|c|c|c|}
\hline $\begin{array}{l}\text { Phase } \\
\text { (in degrees) }\end{array}$ & $\begin{array}{l}\Delta U \\
\text { (Var-Comp) }\end{array}$ & $\begin{array}{l}\text { No. of } \\
\text { points }\end{array}$ & $\begin{array}{l}\text { Phase } \\
\text { (in degrees) }\end{array}$ & $\begin{array}{l}\Delta U \\
\text { (Var-Comp) }\end{array}$ & $\begin{array}{l}\text { No. of } \\
\text { points }\end{array}$ \\
\hline 0.066 & 1.110 & 4 & 75.165 & -0.011 & 6 \\
\hline 1.324 & 1.102 & 4 & 77.390 & 0.010 & 5 \\
\hline 2.247 & 1.132 & 4 & 80.727 & 0.021 & 11 \\
\hline 2.874 & 1.076 & 4 & 83.913 & 0.047 & 10 \\
\hline 4.444 & 1.011 & 4 & 86.911 & 0.029 & 8 \\
\hline 5.196 & 0.951 & 4 & 89.884 & 0.037 & 6 \\
\hline 5.603 & 0.936 & 4 & 92.118 & 0.014 & 5 \\
\hline 6.444 & 0.845 & 4 & 95.988 & 0.041 & 6 \\
\hline 6.852 & 0.850 & 4 & 97.723 & 0.048 & 7 \\
\hline 7.180 & 0.850 & 4 & 102.548 & -0.012 & 8 \\
\hline 8.034 & 0.750 & 4 & 106.149 & 0.039 & 5 \\
\hline 9.020 & 0.657 & 4 & 108.365 & 0.039 & 11 \\
\hline 9.631 & 0.628 & 4 & 111.421 & 0.056 & 10 \\
\hline 10.437 & 0.556 & 4 & 114.750 & 0.046 & 9 \\
\hline 10.926 & 0.543 & 4 & 118.665 & 0.036 & 13 \\
\hline 11.772 & 0.494 & 3 & 122.676 & 0.027 & 12 \\
\hline 12.285 & 0.451 & 4 & 125.779 & 0.055 & 6 \\
\hline 13.402 & 0.391 & 4 & 127.790 & 0.022 & 9 \\
\hline 14.479 & 0.315 & 4 & 131.686 & 0.031 & 6 \\
\hline 14.905 & 0.257 & 3 & 134.458 & 0.064 & 8 \\
\hline 15.662 & 0.328 & 3 & 137.356 & 0.035 & 10 \\
\hline 16.164 & 0.298 & 4 & 141.244 & 0.042 & 10 \\
\hline 16.980 & 0.293 & 4 & 144.893 & 0.048 & 7 \\
\hline 17.673 & 0.181 & 3 & 147.761 & 0.050 & 7 \\
\hline 18.512 & 0.166 & 4 & 150.174 & 0.040 & 5 \\
\hline 19.694 & 0.133 & 4 & 152.162 & 0.053 & 8 \\
\hline 20.028 & 0.118 & 4 & 154.819 & 0.039 & 3 \\
\hline 21.387 & 0.091 & 3 & 157.328 & 0.107 & 3 \\
\hline 22.207 & 0.115 & 3 & 160.586 & 0.105 & 4 \\
\hline 22.577 & 0.082 & 4 & 165.036 & 0.100 & 4 \\
\hline 23.408 & 0.091 & 3 & 166.706 & 0.089 & 3 \\
\hline 23.898 & 0.038 & 4 & 167.139 & 0.152 & 3 \\
\hline 25.523 & 0.048 & 4 & 170.427 & 0.150 & 3 \\
\hline 27.262 & 0.060 & 4 & 171.211 & 0.152 & 3 \\
\hline 29.483 & 0.069 & 11 & 171.771 & 0.160 & 2 \\
\hline 32.628 & 0.046 & 10 & 174.251 & 0.168 & 3 \\
\hline 35.479 & 0.074 & 6 & 175.393 & 0.202 & 3 \\
\hline 37.456 & 0.030 & 6 & 176.286 & 0.163 & 2 \\
\hline 41.759 & 0.046 & 9 & 178.535 & 0.196 & 3 \\
\hline 44.801 & 0.018 & 2 & 179.985 & 0.206 & 3 \\
\hline 47.327 & 0.058 & 13 & 181.934 & 0.182 & 2 \\
\hline 51.591 & 0.048 & 5 & 183.970 & 0.190 & 3. \\
\hline 54.524 & 0.055 & 6 & 185.612 & 0.196 & 4 \\
\hline 57.109 & 0.047 & 6 & 187.723 & 0.180 & 4 \\
\hline 61.427 & 0.007 & 8 & 189.045 & 0.153 & 3 \\
\hline 64.384 & 0.019 & 5 & 189.910 & 0.144 & 2 \\
\hline 67.149 & -0.008 & 7 & 191.675 & 0.199 & 3 \\
\hline 70.980 & 0.012 & 10 & 192.484 & 0.145 & 4 \\
\hline
\end{tabular}


Table Ic (continued)

\begin{tabular}{|c|c|c|c|c|c|}
\hline $\begin{array}{l}\text { Phase } \\
\text { (in degrees) }\end{array}$ & $\begin{array}{l}\Delta U \\
\text { (Var-Comp) }\end{array}$ & $\begin{array}{l}\text { No. of } \\
\text { points }\end{array}$ & $\begin{array}{l}\text { Phase } \\
\text { (in degrees) }\end{array}$ & $\begin{array}{l}\Delta U \\
\text { (Var-Comp) }\end{array}$ & $\begin{array}{l}\text { No. of } \\
\text { points }\end{array}$ \\
\hline 193.722 & 0.121 & 4 & 308.090 & 0.045 & 10 \\
\hline 196.232 & 0.098 & 4 & 311.227 & 0.060 & 7 \\
\hline 197.242 & 0.118 & 4 & 314.112 & 0.061 & 8 \\
\hline 199.820 & 0.038 & 2 & 318.513 & 0.053 & 5 \\
\hline 200.907 & 0.063 & 2 & 320.986 & 0.068 & 7 \\
\hline 201.511 & 0.072 & 3 & 325.875 & 0.083 & 8 \\
\hline 203.177 & 0.064 & 3 & 330.102 & 0.066 & 6 \\
\hline 205.426 & 0.056 & 3 & 333.995 & 0.082 & 4 \\
\hline 206.041 & 0.061 & 3 & 335.919 & 0.086 & 5 \\
\hline 208.340 & 0.068 & 4 & 337.307 & 0.089 & 4 \\
\hline 211.006 & 0.082 & 5 & 338.154 & 0.112 & 4 \\
\hline 213.901 & 0.063 & 5 & 339.882 & 0.134 & 4 \\
\hline 217.483 & 0.064 & 5 & 340.380 & 0.152 & 4 \\
\hline 221.512 & 0.066 & 8 & 340.955 & 0.116 & 3 \\
\hline 225.575 & 0.045 & 9 & 342.534 & 0.224 & 3 \\
\hline 233.642 & 0.017 & 10 & 342.817 & 0.201 & 3 \\
\hline 239.205 & 0.021 & 6 & 343.729 & 0.266 & 4 \\
\hline 243.193 & 0.023 & 8 & 344.178 & 0.242 & 5 \\
\hline 248.524 & 0.011 & 8 & 344.618 & 0.248 & 4 \\
\hline 254.073 & 0.021 & 9 & 344.998 & 0.075 & 4 \\
\hline 257.624 & 0.013 & 11 & 346.489 & 0.391 & 4 \\
\hline 260.492 & 0.006 & 11 & 347.042 & 0.416 & 4 \\
\hline 264.450 & 0.003 & 11 & 347.498 & 0.400 & 3 \\
\hline 267.771 & -0.001 & 9 & 348.466 & 0.452 & 4 \\
\hline 270.206 & -0.020 & 5 & 348.793 & 0.429 & 4 \\
\hline 273.773 & -0.013 & 9 & 349.241 & 0.465 & 4 \\
\hline 276.323 & 0.005 & 11 & 351.399 & 0.656 & 4 \\
\hline 280.075 & 0.000 & 10 & 352.593 & 0.729 & 5 \\
\hline 283.243 & 0.016 & 15 & 353.066 & 0.768 & 3 \\
\hline 286.607 & -0.007 & 10 & 353.606 & 0.800 & 4 \\
\hline 289.406 & 0.010 & 10 & 354.463 & 0.852 & 3 \\
\hline 292.372 & 0.009 & 12 & 355.603 & 1.000 & 4 \\
\hline 296.023 & 0.025 & 13 & 356.307 & 1.071 & 3 \\
\hline 298.154 & 0.034 & 6 & 356.978 & 1.062 & 4 \\
\hline 301.165 & 0.052 & 11 & 357.656 & 1.083 & 4 \\
\hline \multirow[t]{2}{*}{305.266} & 0.040 & 9 & 358.262 & 1.097 & 3 \\
\hline & & & 359.106 & 1.142 & 4 \\
\hline
\end{tabular}

method of least squares and the derived coefficients are given in Table II. For rectification of light, the $\cos 3 \theta, \cos 4 \theta$, and all the sine terms representing the light were substracted from the wave corrected light.

The reflection coefficients $C_{0}, C_{1}$, and $C_{2}$ (given in Table II) were obtained using the following equations (Russell and Merrill, 1952):

$$
\begin{aligned}
& C_{0}=-\left(0.75-0.25 \cos ^{2} i\right) \frac{G_{c}+G_{h}}{G_{c}-G_{h}} A_{1} \operatorname{cosec} i, \\
& C_{1}=-A_{1},
\end{aligned}
$$




$$
C_{2}=-0.25 \frac{G_{c}+G_{h}}{G_{c}-G_{h}} A_{1} \sin i \text {. }
$$

The $G_{c}$ and $G_{h}$ values used in the above equations were obtained from Cester's (1969) tables for the spectral types (G5V, K1V) of the individual components and an inclination (i) equal to $88^{\circ} .5$ was used. The coefficients thus derived were used in the equation

$$
N z=\frac{-4\left(A_{2}-C_{2}\right)}{\left(A_{0}-C_{0}\right)-\left(A_{2}-C_{2}\right)}
$$

to compute the values of $z$. The values of $N$ used in the above equation and given in Table III were taken from Princeton contribution No. 26 except for the value which represents the cooler component in the ultraviolet passband. In this case the value of $N$ was estimated using the following equation given by Russell and Merrill

TABLE II

UV Psc: Fourier and rectification coefficients

\begin{tabular}{lccc}
\hline & $V$ & $B$ & $U$ \\
\hline$A_{0}$ & +0.9874 & +0.9629 & +0.9671 \\
& \pm 0.0001 & \pm 0.0001 & \pm 0.0001 \\
$A_{1}$ & -0.0042 & -0.0027 & -0.0069 \\
& \pm 0.0001 & \pm 0.0001 & \pm 0.0001 \\
$A_{2}$ & -0.0292 & -0.0263 & -0.0306 \\
& \pm 0.0001 & \pm 0.0001 & \pm 0.0001 \\
$A_{3}$ & -0.0008 & -0.0012 & -0.0050 \\
& \pm 0.0001 & \pm 0.0001 & \pm 0.0001 \\
$A_{4}$ & -0.0056 & +0.0012 & +0.0013 \\
& \pm 0.0001 & \pm 0.0001 & \pm 0.0001 \\
$B_{1}$ & -0.0017 & -0.0009 & -0.0022 \\
& \pm 0.0001 & \pm 0.0001 & \pm 0.0001 \\
$B_{2}$ & -0.0010 & -0.0012 & +0.0015 \\
& \pm 0.0001 & \pm 0.0001 & \pm 0.0001 \\
$B_{3}$ & +0.0064 & +0.0079 & +0.0100 \\
& \pm 0.0001 & \pm 0.0001 & \pm 0.0001 \\
$B_{4}$ & -0.0046 & -0.0022 & -0.0036 \\
& \pm 0.0001 & \pm 0.0001 & \pm 0.0001 \\
$C_{0}$ & +0.0116 & +0.0102 & +0.0462 \\
$C_{1}$ & +0.0042 & +0.0027 & +0.0069 \\
$C_{2}$ & +0.0039 & +0.0034 & +0.0154 \\
$z$ & 0.045 & 0.038 & 0.049 \\
\hline & & &
\end{tabular}


(1952):

$$
N=\frac{(15+x)(1+y)}{15-5 x}
$$

where $x$ (limb darkening coefficient $)=1.0$ and $y$ (gravity darkening coefficient $)=2.012$ were used (Kopal, 1959), Table 4-5).

TABLE III

UV Psc: adopted values of ' $x$ ' and ' $N$ ' for $U B V$ colours

\begin{tabular}{llll}
\hline Colour & Star & $x$ & $N$ \\
\hline$V$ & hotter component & 0.6 & 2.6 \\
& cooler component & 0.8 & 3.2 \\
$B$ & hotter component & 0.8 & 3.2 \\
& cooler component & 0.8 & 3.2 \\
$U$ & hotter component & 0.8 & 3.2 \\
& cooler component & 1.0 & 4.8 \\
\hline
\end{tabular}

The phase angle $\theta$ was rectified using the equation

$$
\sin ^{2} \Theta=\frac{\sin ^{2} \theta}{1-z \cos ^{2} \theta}
$$

where $z=0.044$ (an average value for all $U B V$ passbands). The light curves were then rectified using the formula given below and the coefficients given in Table II.

$$
\begin{aligned}
l_{\text {rec }}^{\text {cor }}= & {\left[l^{\text {cor }}+C_{0}+C_{1} \cos \theta+C_{2} \cos 2 \theta-\sum_{n=1}^{4} B_{n} \sin n \theta-\right.} \\
& \left.-\sum_{n=3}^{4} A_{n} \cos n \theta\right] /\left[\left(A_{0}+C_{0}\right)+\left(A_{2}+C_{2}\right) \cos 2 \theta\right] .
\end{aligned}
$$

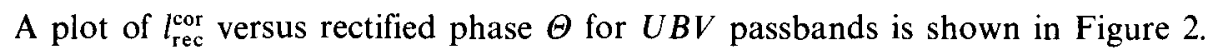

\section{Intrinsic Variation}

\subsection{SOURCE OF THE INTRINSIC VARIATION}

A close inspection of Figures 1a-1i (Vivekananda Rao and Sarma, 1981b) show that outside of the eclipses, the observations have a scatter of \pm 0.05 which is larger than the estimated internal probable error $( \pm 0.02)$. Such a large scatter was also found in some other RS CVn type binaries like SS Cam (Arnold et al., 1979), TY Pyx (Vivekananda Rao and Sarma, 1981a), and AR Lac (Chambliss, 1976). 


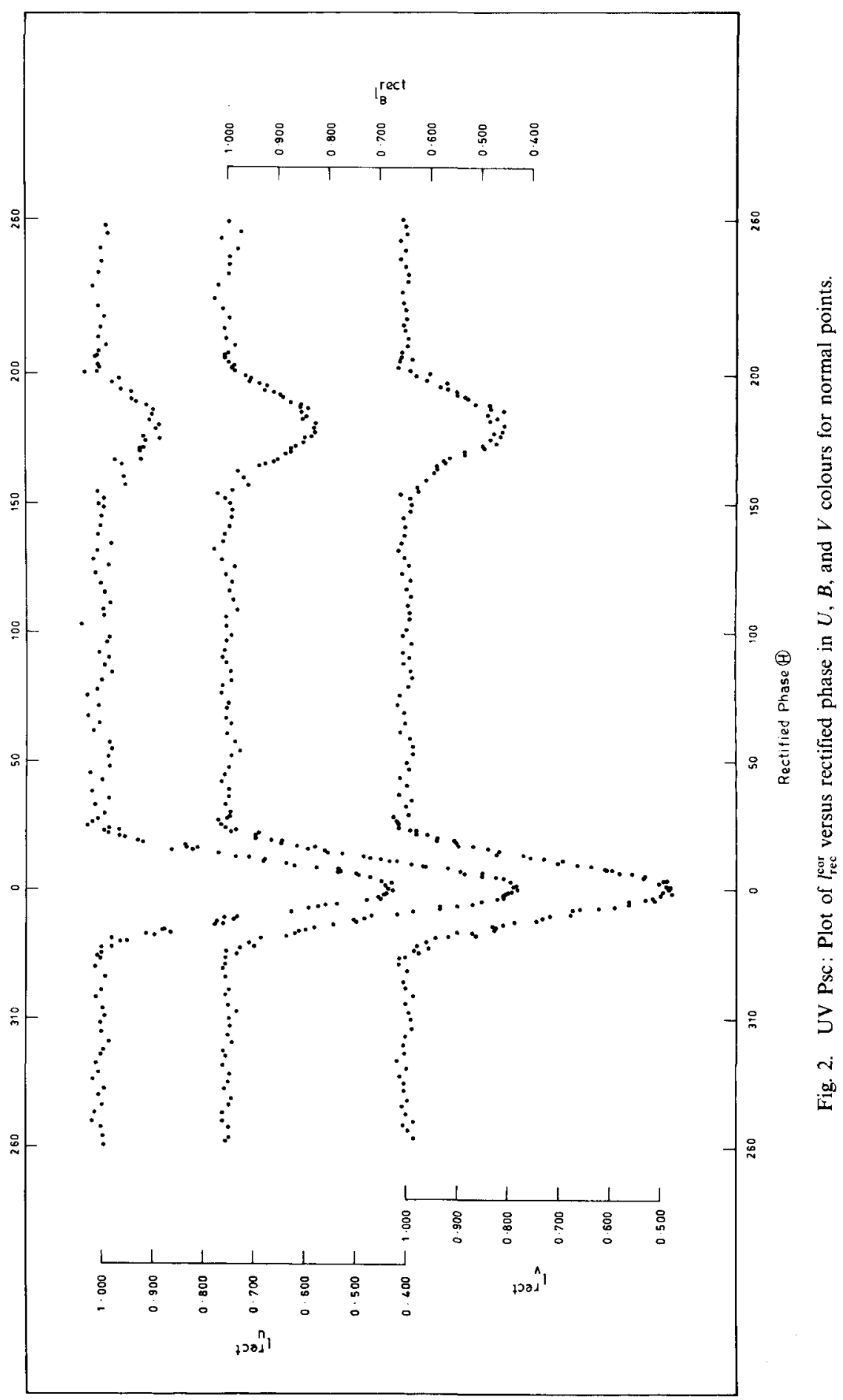


This variation has been attributed to the intrinsic variability of one or both components of the system. Hence, it is possible that the large scatter observed in the light curves of UV Psc could be due to the intrinsic variability of its component(s). In order to detect which component(s) of the system is responsible for the observed intrinsic variation all the rectified individual observations outside the eclipses were used. The residuals $\left(1-l_{\text {rec }}^{\text {cor }}\right)=\delta l_{i}$ were found to be larger than the observational errors and the range of the nightly means of the residuals are given below.

$$
\begin{array}{lll}
\delta l_{V}=-0.042 \pm 0.005 & \text { to } & +0.033 \pm 0.003 \\
\delta l_{B}=-0.032 \pm 0.004 & \text { to } & +0.046 \pm 0.007 \\
\delta l_{U}=-0.047 \pm 0.004 & \text { to } & +0.058 \pm 0.004
\end{array}
$$

The following methods were used to detect the component responsible for this intrinsic variation.

(i) The largest and best defined residuals were noticed on two nights: JD 2443490.5 (where the residuals showed a systematic trend) and JD 2443861.5 (where the residuals did not show any systematic trend). These residuals are shown in Figures $3 \mathrm{a}$ and $3 \mathrm{~b}$. The mean values of these residuals are given below:

$$
\begin{aligned}
& \text { JD 2443490.5: } \quad \delta l_{V}=-0.026 \pm 0.004 \text {, } \\
& \delta l_{B}=-0.026 \pm 0.004 \text {, } \\
& \delta l_{U}=-0.032 \pm 0.008 \text {, } \\
& \text { JD 2443861.5: } \quad \delta l_{V}=+0.038 \pm 0.003 \text {, } \\
& \delta l_{B}=+0.037 \pm 0.003 \text {, } \\
& \delta l_{U}=+0.058 \pm 0.003 \text {. }
\end{aligned}
$$

In order to estimate the colour of the residuals, we have computed the percentages of these variations in terms of the hotter and cooler star's light in all passbands and are given in Table IV. In calculating these percentages the $L_{h}$ and $L_{c}$ values given in Table $\mathrm{V}$ were used.

TABLE IV

UV Psc: percentage of the intrinsic variations in terms of $L_{h}$ and $L_{c}$

\begin{tabular}{llrrr}
\hline Date & Star & \multicolumn{1}{c}{$\boldsymbol{V}$} & \multicolumn{1}{c}{$B$} \\
\hline JD 2443490.5 & hotter star & $3.2 \pm 0.5$ & $3.1 \pm 0.5$ & $3.6 \pm 0.7$ \\
& cooler star & $14.2 \pm 2.2$ & $16.1 \pm 2.5$ & $30.5 \pm 7.6$ \\
& & & & \\
JD 2443861.5 & hotter star & $4.0 \pm 0.4$ & $4.4 \pm 0.4$ & $6.5 \pm 0.4$ \\
& cooler star & $18.1 \pm 1.6$ & $23.0 \pm 1.8$ & $55.2 \pm 2.8$ \\
\hline
\end{tabular}




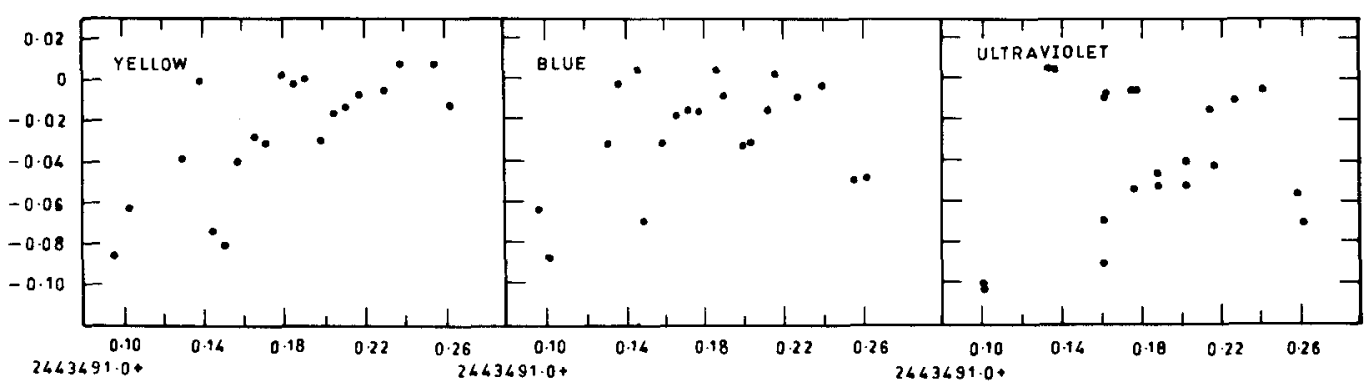

Heliocentric Julian Day

Fig. 3a. UV Psc: Plot of $(\mathrm{O}-\mathrm{C})$ in light versus heliocentric Julian Day for $U B V$ colours. The (O-C) represents the residual between the rectified observed points outside the eclipse and the computed light (equal to unity). A systematic trend can be seen in this plot.

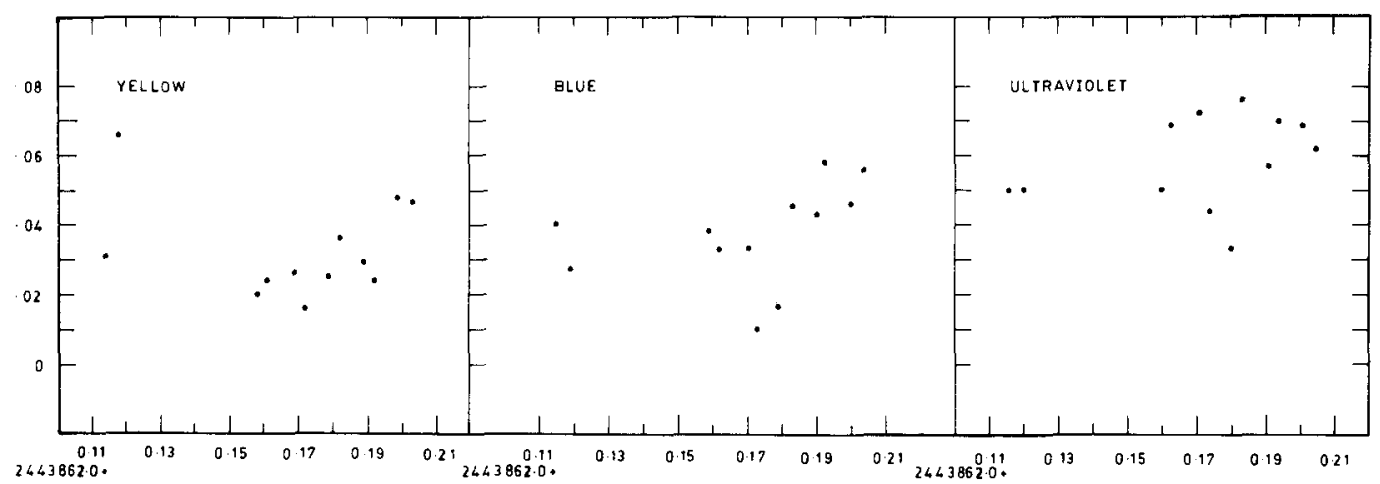

Heliocentric Julian Day

Fig. 3b. UV Psc: Same as Figure 3a for another day where no systematic trend is found.

It is very clear from Table IV that these residuals have nearly the same percentage of the hotter star's light in all the three passbands and on both the nights.

(ii) Taking mean residuals on all the nights except those in eclipses, we have pplotted $\delta l_{V}$ versus $\delta l_{B}$ and also $\delta l_{U}$ versus $\delta l_{U}$ in Figures $4 \mathrm{a}$ and $4 \mathrm{~b}$. The first figure gave a slope of $\left(\delta l_{B} / \delta l_{V}\right)=1.15$ and it is expected to have a slope of 1.02 if the hotter (G5) component is varying whereas a value of 0.78 is expected if the cooler (K1) star is varying. The second figure gave a slope of $\delta l_{U} / \delta l_{V}=1.20$ and a value of 0.80 is expected if the hotter component is variable and a value of 0.54 if the cooler component is variable. Thus, the slopes obtained here are in agreement with the values expected if the hotter component is varying and hence we conclude that the hotter star is responsible for the intrinsic variation. 

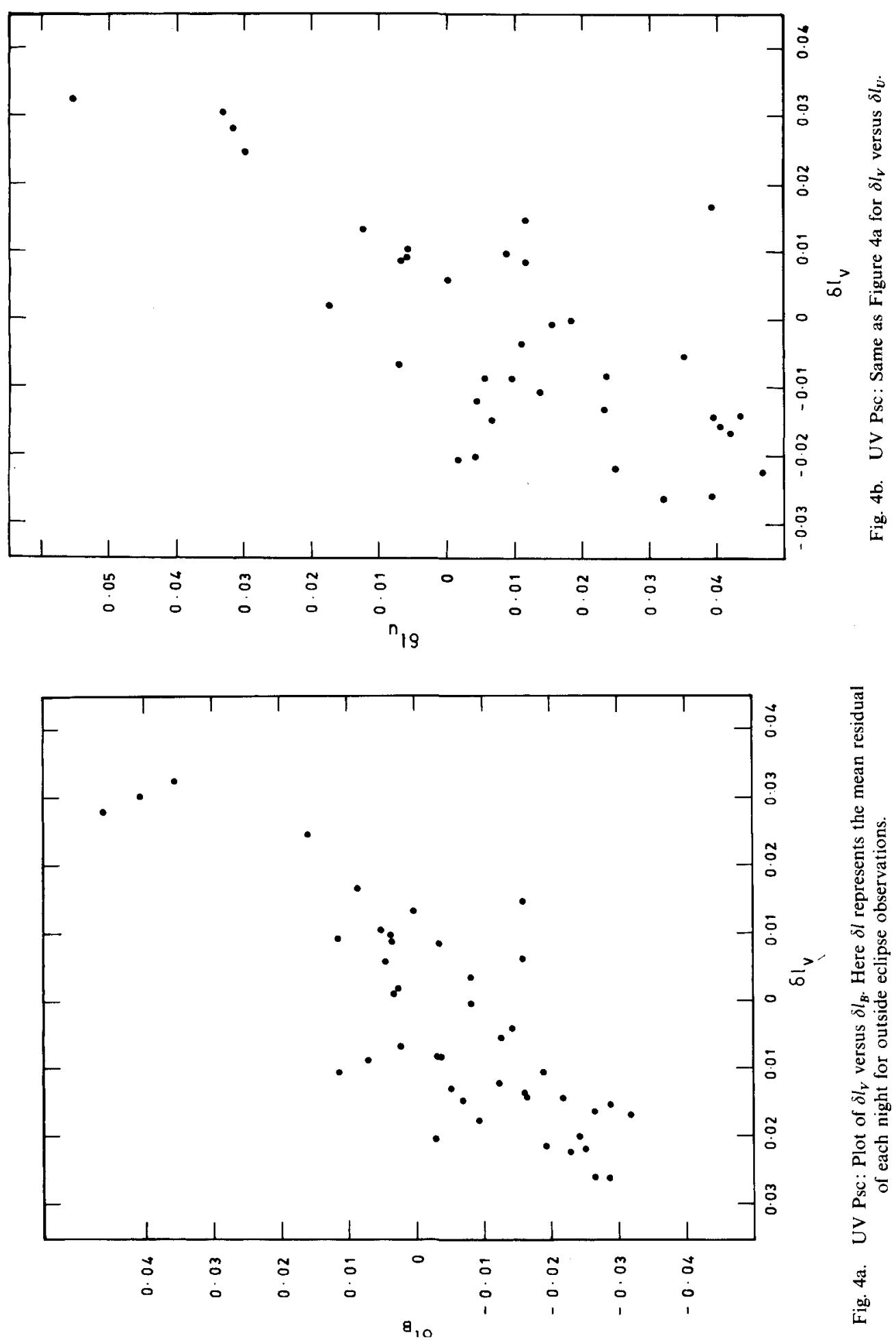


\subsection{Periodicity of the intrinsic Variation}

Individual residuals within each night were examined carefully to determine periodic nature of the variability. The residuals were found to be more or less constant during a night without any significant trend except the night of JD 2443490.5. Even an examination of the residuals over several nights did not yield any trend or periodicity except for a random distribution of the residuals. Since no periodicity could be established for these variations, it is not possible to remove its effect from the eclipses data. This will not have any significant effect on the solution of the primary and secondary eclipses for computation of elements as the percentage of contribution of these variations is only about $5 \%$ of the total light and in addition, due to averaging of data from large number of nights, the net effect is expected to be negligible.

\section{Solution}

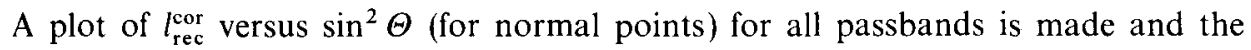
appearance of the light curves suggests that the eclipses are total. Further as $\chi_{0.8}^{\mathrm{sec}}>\chi_{0.8}^{\mathrm{pr}}$ the primary eclipse is found to be a transit and secondary an occultation. This conclusion is in agreement with that of Carr (1969) and Sadik (1979). Modified Wellmann's method was used to solve the light curves in all passbands and the details of this method were given by Vivekananda Rao and Sarma (1981a). Tsesevich (1940) tables were used to read the ' $p$ ' values for the assumed limb darkening coefficients of $x_{h}=0.6$ and $x_{c}=0.8$ in $V$ passband; $x_{h}=x_{c}=0.8$ in $B$ passband; and $x_{h}=0.8$ and $x_{c}=1.0$ in $U$ passband. As the solution of primary eclipse alone and primary plus secondary eclipse combined together yielded about the same value for $\sin ^{2} \Theta_{e}$, the data for both primary and secondary eclipses together were used to derive $\sin ^{2} \Theta_{e}$ for different ' $k$ ' values. But in the case of $U$ light curve, due to very small depth of the secondary eclipse, only primary eclipse was used to derive the value of $\sin ^{2} \Theta_{e}$. Figures $5 \mathrm{a}, 5 \mathrm{~b}$, and $5 \mathrm{c}$ show the plot of $\sum w\left(l_{0}-l_{c}\right)^{2}$ against $k$ for $V B U$ passbands. A weighted average of all the three passbands gave $k=0.75 \pm 0.01$. This value of $k$ agrees quite well with the value of $k=0.75$ determined by Carr (1969) and Oliver (1974) and $k=0.762$ determined by Sadik (1979). Corrections for the depths (primary and secondary) of the hand drawn curve were obtained according to the modified Wellmann's method. These corrections were small ( $\sim 0.003$ in luminosity units) in $B$ and $V$ passbands and gave the same value for the geometric depth. But in the case of ultraviolet light curve, the correction amounted to a large value $(\sim 0.04$ in luminosity units) which the observations did not permit. Hence, no depth correction could be applied to the $U$ light curve. Since both $B$ and $V$ light curves gave an unique solution, elements were computed using these curves for $k=0.75$. The averaged elements for the system are given in Table V. Using these elements, theoretical curves were computed for $V B U$ passbands and Figures 6a-6f show the 

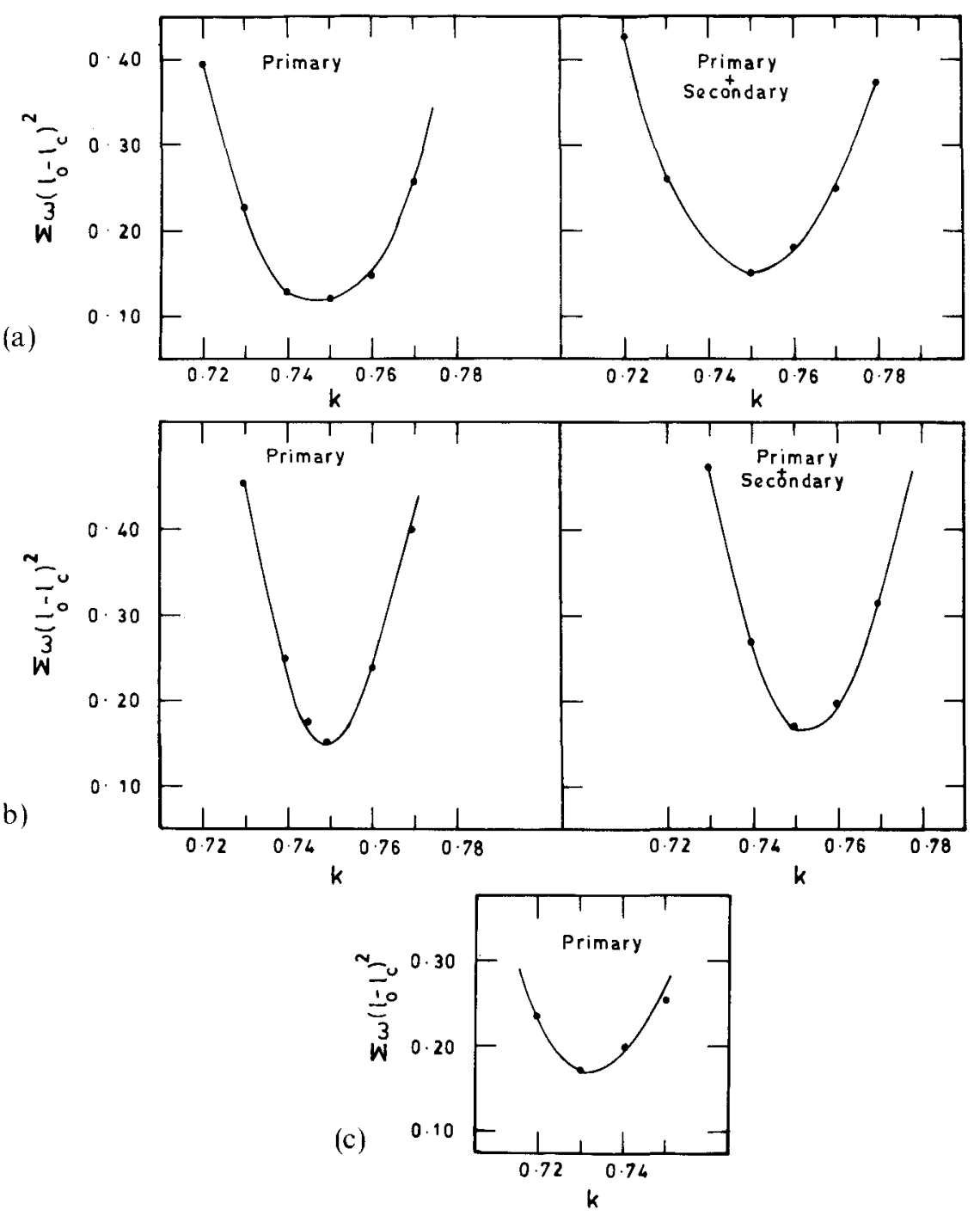

Fig. 5. UV Psc: Plot $\sum w\left(l_{0}-l_{c}\right)^{2}$ versus $k$ for primary and secondary minima. (a) in yellow colour (b) in blue colour and (c) ultraviolet colour (primary only).

plot of the observed normals and the theoretical fit (solid line). The fit of the theoretical curves to the observed normal points in all passbands (in primary and secondary) is found to be quite satisfactory.

The absolute radii $R_{h, c}$ for the two components were estimated from the relationship

$$
m_{h}+m_{c}=\frac{1}{74.55} \frac{1}{P^{2}}\left(\frac{R_{h, c}}{r_{h, c}}\right)^{3},
$$

where $P$ is the orbital period in days, $r_{h, c}$ are the fractional radii of individual 


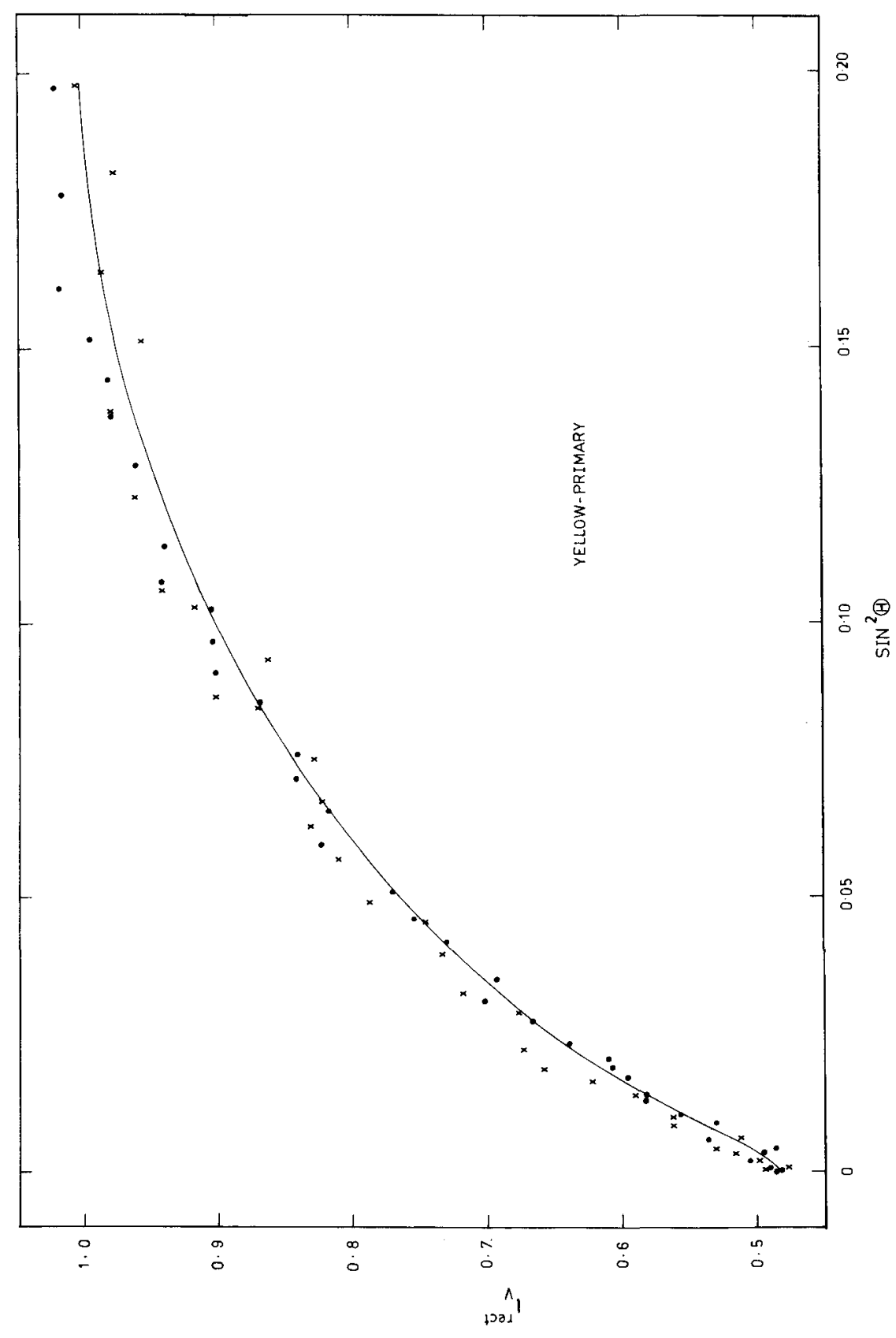



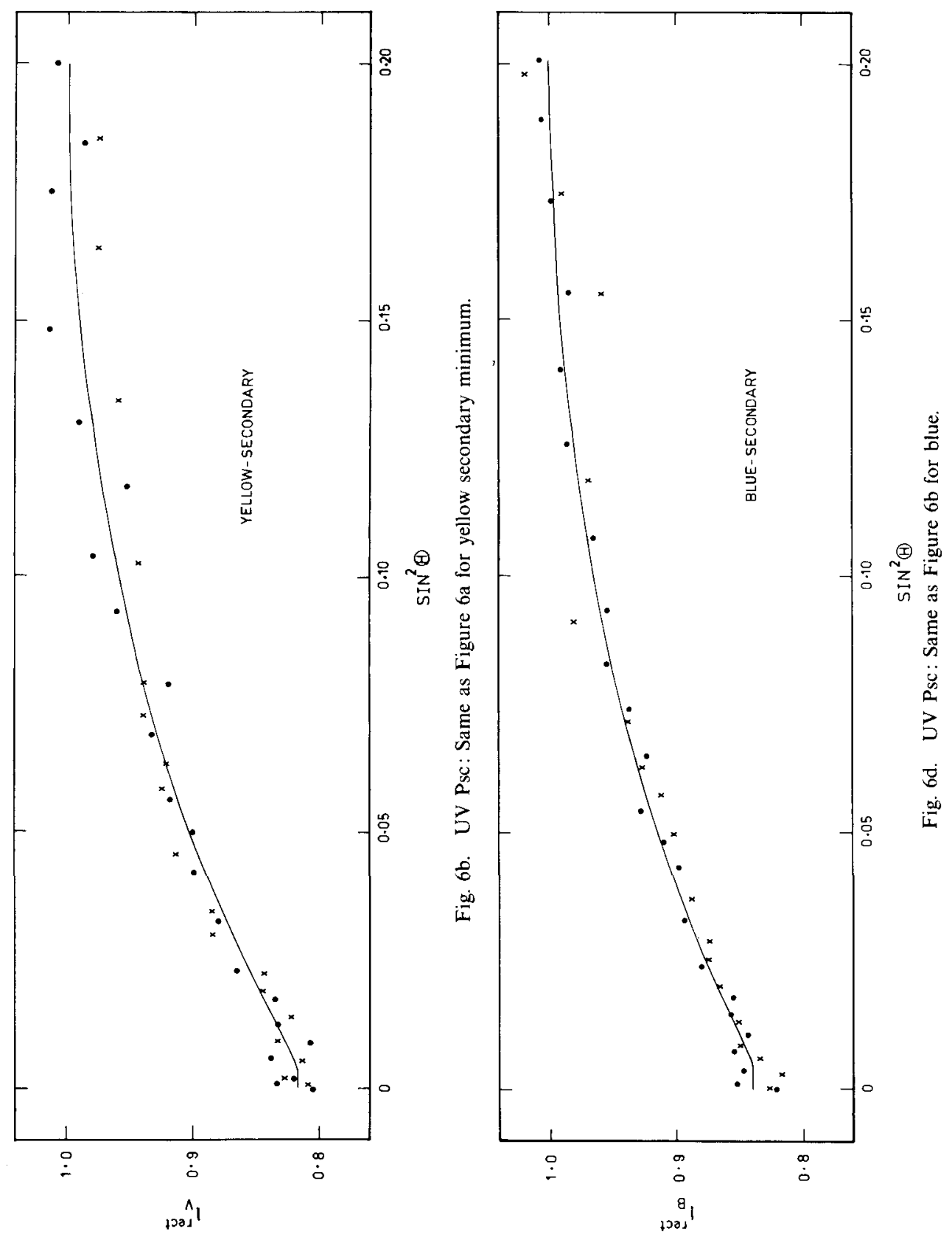


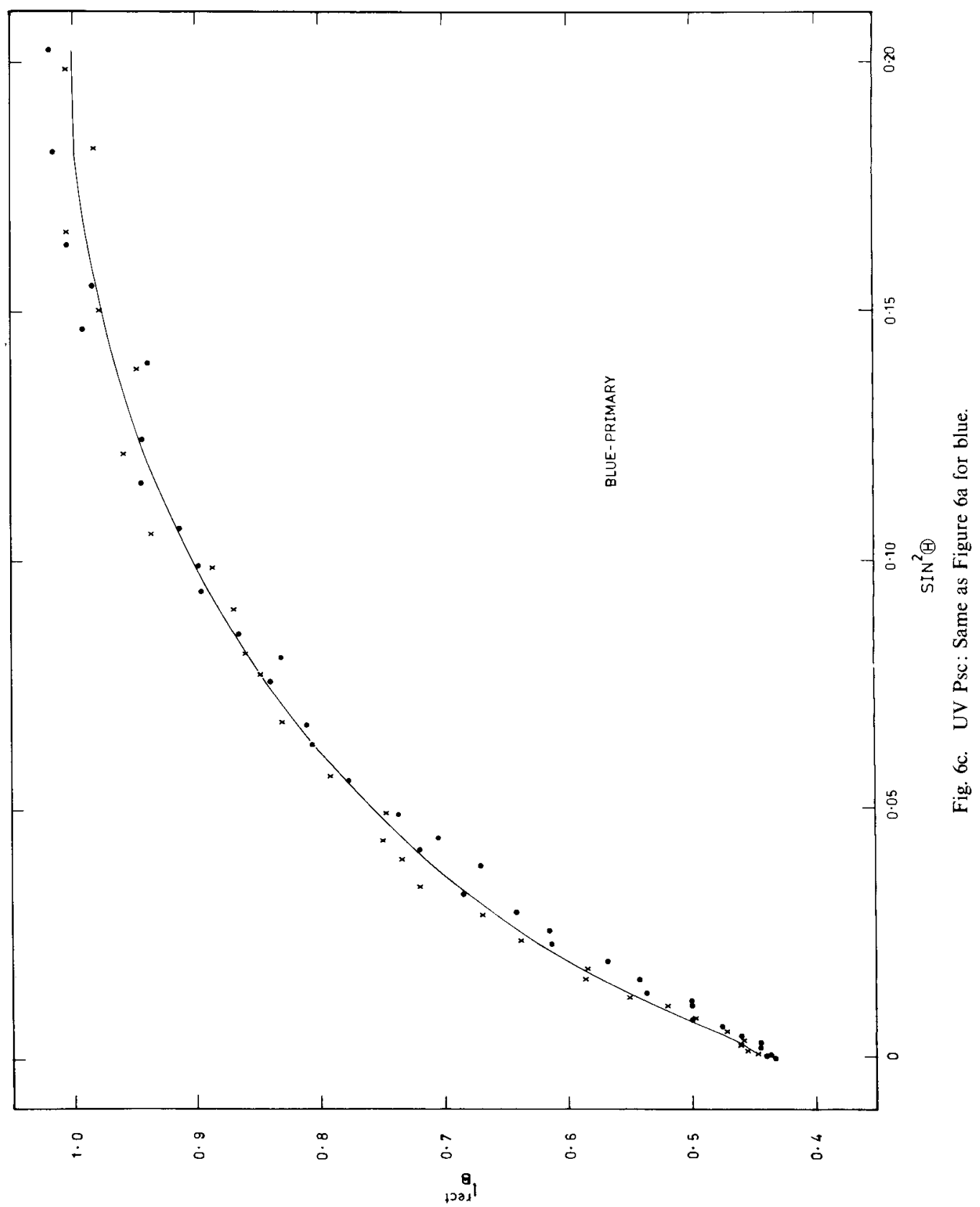




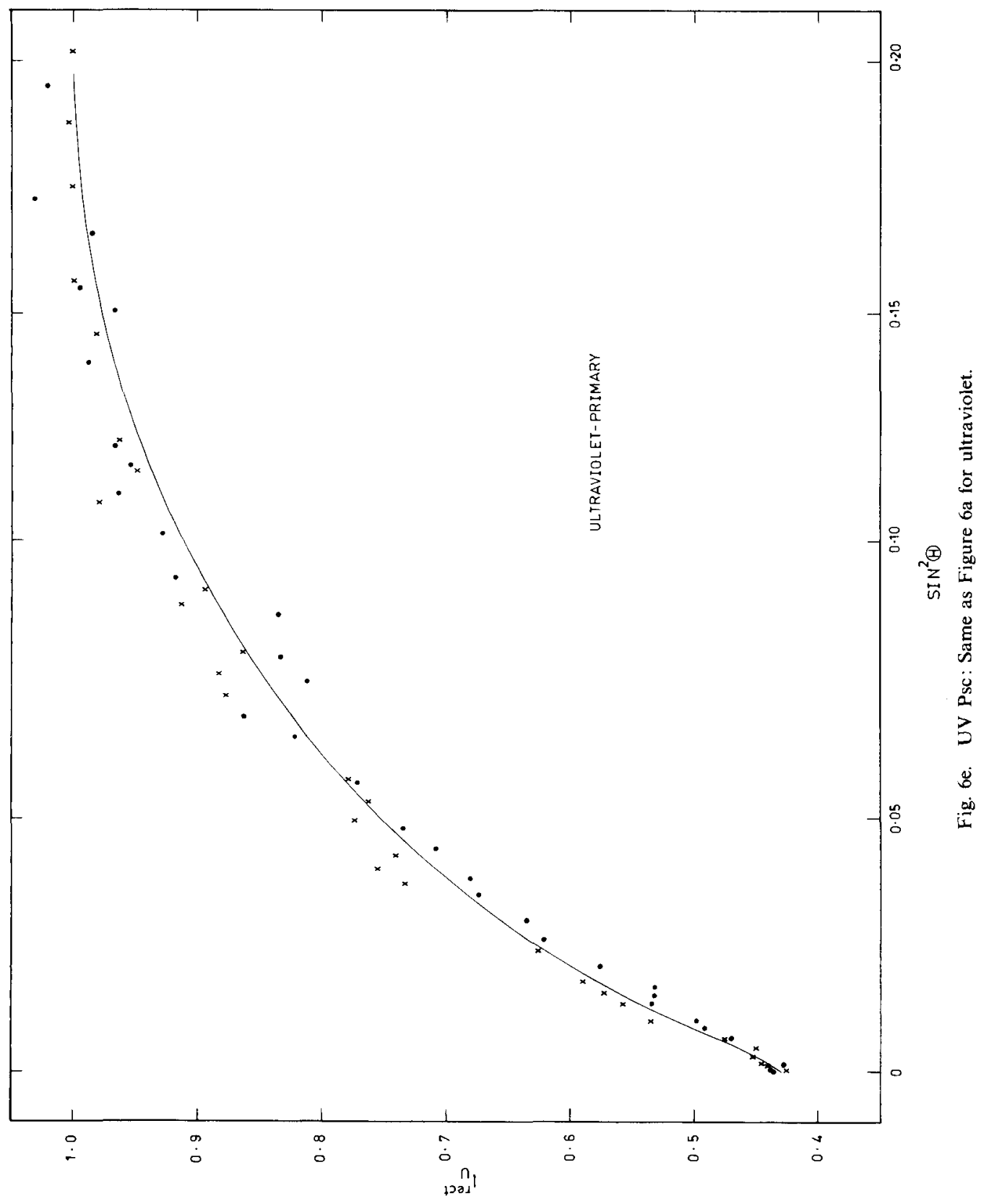




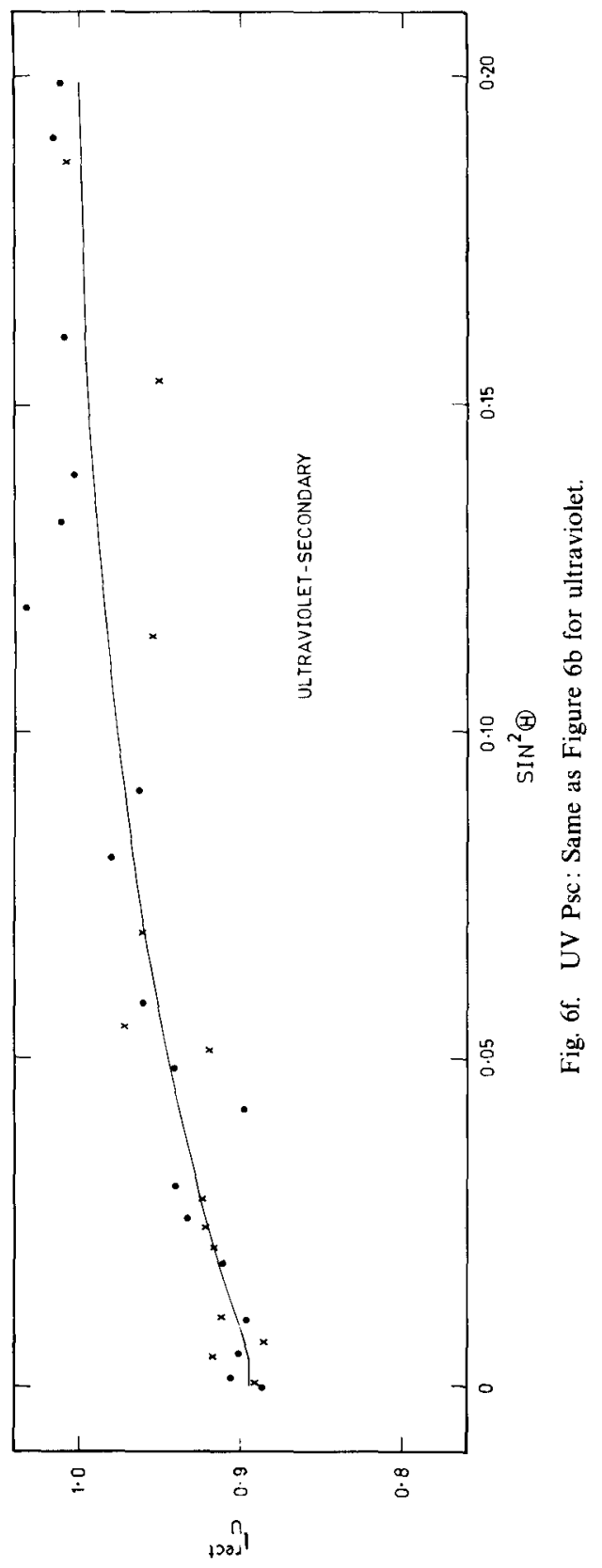


components, and $m_{h}$ and $m_{c}$ are the individual masses of the components. Popper (1976) had estimated the masses as $1.20 \mathrm{~m}_{\odot}$ and $0.90 \mathrm{~m}_{\odot}$ for the hotter and cooler components, respectively. Thus, the estimated radius of the hotter and cooler components of UV Psc will be $1.24 \pm 0.01 R_{\odot}$ and $0.93 \pm 0.01 R_{\odot}$, respectively.

TABLE V

Adopted elements for UV Psc

\begin{tabular}{llll}
\hline Element & $V$ & $B$ & $U$ \\
\hline$x_{h}$ & 0.6 & 0.8 & 0.8 \\
$x_{c}$ & 0.8 & 0.8 & 1.0 \\
$\alpha_{0}^{\text {tr }}$ & 1.030 & 1.043 & 1.043 \\
$\alpha_{0}^{\text {oc }}$ & 1.000 & 1.000 & 1.000 \\
$1-l_{0}^{\text {tr }}$ & 0.518 & 0.559 & 0.570 \\
$1-l_{0}^{\text {oc }}$ & 0.182 & 0.161 & 0.105 \\
$L_{h}$ & 0.818 & 0.839 & 0.895 \\
$L_{c}$ & 0.182 & 0.161 & 0.105 \\
$J_{h} / J_{c}$ & 2.54 & 2.95 & 4.59 \\
$L_{h}^{\prime \prime}$ & 0.831 & 0.851 & 0.905 \\
$L_{c}^{\prime \prime}$ & 0.169 & 0.149 & 0.095 \\
$J_{h}^{\prime \prime} / J_{c}^{\prime \prime}$ & 2.77 & 3.21 & 5.36 \\
$\Theta_{e}$ & & 26.390 & \\
$p_{0}$ & & -1.20 & \\
$k$ & & $0.75 \pm 0.01$ & \\
$j$ & & $88.53 \pm 0.5$ & \\
$r_{h}=a_{h}$ & & $0.254 \pm 0.002$ & \\
$r_{c}=a_{c}$ & & $0.191 \pm 0.002$ & \\
$b_{h}$ & & $0.248 \pm 0.002$ & \\
$b_{c}$ & & $0.187 \pm 0.002$ & \\
\hline
\end{tabular}

\section{Discussion}

By use of the values

$$
\begin{aligned}
& V=9^{m} .62 \pm 0.02 \\
& B-V=+0^{m} .51 \pm 0.01 \\
& U-B=-0^{m} .01 \pm 0.02
\end{aligned}
$$

for the comparison star and the differential magnitudes corresponding to unit luminosity at maximum light, a value of $V=9^{m} \cdot 12 \pm 0.02, B=9^{m} .87 \pm 0.02$, and $U=10^{m} .13 \pm 0.02$ for the variable at maximum light were obtained. The luminosities of the individual components corrected for the reflected light were obtained from $L_{h}$ and $L_{c}$ (given in Table V) according to the equation of Koch et al. (1970)

$$
\left.\begin{array}{l}
L_{h}^{\prime}=L_{h}-0.8 L_{c}(a b)_{h} E_{h} / E_{c}, \\
L_{c}^{\prime}=L_{c}-0.8 L_{h}(a b)_{c} E_{c} / E_{h},
\end{array}\right\}
$$


where the values of $E_{h}$ and $E_{c}$ were taken from Cester's (1969) tables. The results of this calculation applied to the luminosity in $V, B$, and $U$ are given in Table $\mathrm{V}$ as $L_{h}^{\prime \prime}$ and $L_{c}^{\prime \prime}$ after normalization. From these values the magnitudes and colours for the two components were obtained.

\begin{tabular}{llr}
\hline & hotter & \multicolumn{1}{c}{ cooler } \\
\hline$V$ & $9^{m} \cdot 32 \pm 0.02$ & $11^{m} \cdot 05 \pm 0.02$ \\
$B-V$ & $0^{m} \cdot 72 \pm 0.01$ & $0^{m} \cdot 89 \pm 0.01$ \\
$U-B$ & $0^{m} \cdot 20 \pm 0.02$ & $0^{m} \cdot 74 \pm 0.02$ \\
\hline
\end{tabular}

Assuming that the interstellar reddening is negligible, the colours obtained above suggest a spectral type of G4-6 $\left(T_{e}=5520 \pm 100 \mathrm{~K}\right)$ for the hotter component and $\mathrm{K} 0-2\left(T_{e}=4740 \pm 100 \mathrm{~K}\right)$ for the cooler component (Allen, 1976). These spectral types are in agreement with those derived by Carr (1969), Oliver (1974), and Sadik (1979). The spectral type of the cooler component can also be estimated by assuming that the radiation of both the hotter and cooler components may be approximated by the Planck's function over the passbands of $U, B$, and $V$ filters (Wood, 1971). Assuming a temperature of $T_{e}=5520 \mathrm{~K}$ for the hotter component of spectral type $\mathrm{G} 5$, we got a temperature $T_{e}=4654 \mathrm{~K}$ in $V, T_{e}=4700 \mathrm{~K}$ in $B$, and $T_{e}=4525 \mathrm{~K}$ in $U$ for the cooler component with an uncertainty of $\pm 100 \mathrm{~K}$. The average temperature $T_{e}=4626 \pm 100 \mathrm{~K}$ for the cooler component corresponds to a spectral type of K0-2. Thus as the spectral types of the components derived from the above method and also estimated from the colours closely agree with one another, a spectral type of G4-6 $\left(T_{e}=5520 \pm 100 \mathrm{~K}\right)$ for the hotter component and $\mathrm{K} 0-2\left(T_{e}=4740 \pm 100 \mathrm{~K}\right)$ for the cooler component is justified. This consclusion is in agreement with the results of Sadik (1979) who has estimated a temperature of $T_{e}=5740 \mathrm{~K}$ and $T_{e}=4750 \mathrm{~K}$ for the hotter and cooler components, respectively. From the values of temperature and radii given above for each component the bolometric luminosities estimated from Stefan-Boltzmann's law are

$$
\left.\begin{array}{l}
\log \left(L_{h} / L_{\odot}\right)=+0.10 \pm 0.10 \\
\log \left(L_{c} / L_{\odot}\right)=-0.41 \pm 0.11
\end{array}\right\}
$$

for the hotter and cooler components, respectively. From these luminosities, the absolute bolometric magnitudes are determined using

$$
M_{\text {bol }}=4.75-2.5 \log \left(L / L_{\odot}\right)
$$

and these values are

$$
\left.\begin{array}{l}
M_{\text {bol }}(\text { hotter })=+4^{m} \cdot 50 \pm 0.25, \\
M_{\text {bol }}(\text { cooler })=+5^{m} \cdot 83 \pm 0.28 .
\end{array}\right\}
$$


Applying standard bolometric correction of $-0^{m} .07$ and $-0^{m} .27$ for the Main Sequence stars of spectral type $\mathrm{G} 5$ and $\mathrm{K} 1$ the absolute visual magnitudes determined for the two components are

$$
\begin{aligned}
& M_{V}(\text { hotter })=+4^{m} \cdot 57 \pm 0.25, \\
& M_{V}(\text { cooler })=+6^{m} \cdot 10 \pm 0.28
\end{aligned}
$$

These absolute visual magnitudes are in good agreement with the values of $M_{V}$ determined from the photometric distance of $0^{\prime \prime} .012 \pm 0.006\left[M_{v}=(\right.$ hotter $)=$ $=+4^{m} .72 \pm 0.86 M_{V}$ (cooler) $\left.=+6^{m} .45 \pm 0.86\right]$ given by Dworak (1973). The estimated distance obtained from these magnitudes is of the order of 85 parsec and hence our assumption of no interstellar reddening is quite justified. The absolute visual magnitudes given by Allen (1976) (1976) for the corresponding spectral types of G5V and $\mathrm{K} 1 \mathrm{~V}$ are $M_{V}$ (hotter $)=+5^{m} \cdot 1$ and $M_{V}$ (cooler) $=6^{m} \cdot 2$, respectively.

Assuming the hotter and cooler components to be of spectral type and luminosity class as $\mathrm{G} 4-6 \mathrm{~V}$ and $\mathrm{K} 0-2 \mathrm{~V}$, a mass ratio of $m_{2} / m_{1}=0.80 \pm 0.04$ was obtained from Allen (1976). This value of mass ratio is in close agreement with the value of $m_{2} / m_{1}=0.75$, given by Popper (1976) from his spectroscopic studies. From Plavec and Kratochvil's (1964) tables, for a mass ratio of 0.80 , the sizes of the Roche lobes are found to be $r_{h}^{*}=0.395$ and $r_{c}^{*}=0.354$. Comparing these values with $b_{h}=0.248$ and $b_{c}=0.187$ obtained in the present investigation (Table V), we conclude that both the components of UV Psc are well within their Roche lobes suggesting that UV Psc is a detached system like other members of the RS CVn group.

The Main-Sequence luminosity class derived for both the components of UV Psc in the present investigation agrees with that of Carr (1969) but differs from that of Oliver (1974) and Sadik (1979). Oliver (1974) had obtained the primary eclipse to be an occultation and the secondary to be transit and had determined the spectral types and luminosity classes as G2V or G2IV with ultraviolet excess for the hotter component and KOIV for the cooler component. But, the colours obtained from our investigation suggest that the hotter component must be a normal Main Sequence star of spectral type G5V rather than a subgiant with UVexcess as suggested by Oliver (1974). Next, the nature of the primary eclipse as occultation rather than a transit made Oliver (1974) to conclude that the cooler component should be a subgiant. Sadik (1979) concluded the primary eclipse to be a transit and the secondary an occultation and suggested that the spectral type and the luminosity class for the hotter and cooler components as G2V and K0IV, respectively. In such a case, the cooler KOIV star will have a radius larger than the hotter G2V component and hence, the primary eclipse will be an occulation rather than a transit which is contrary to that of his own solution. If the primary eclipse is a transit as obtained by Carr (1969), Sadik (1979) and the present investigation, the cooler component must have a radius smaller than that of the hotter star. This can happen only if the cooler component of UV Psc is a Main Sequence star 
rather than a subgiant. Hence, we suggest that both the components of UV Psc have to be of Main Sequence stars of spectral types G5 and K1 in order to explain the nature of the primary and secondary eclipses. Further, if the cooler component of UV Psc is a subgaint (K0-IV) as suggested by both Oliver (1974) and Sadik (1979) and has to share the properties of other RS CVn candidates like Z Her (Popper, 1956) RS CVn (Popper, 1961), AR Lac (Chambliss, 1976), SZ Psc (Jakate et al., 1976), snd WW Dra (Mardirossian et al., 1980), then its radius should be in the order of around $2.5 R_{\odot}$. However, a radius $\left(R_{c}\right)$ of $0.93 R_{\odot}$ obtained in the present investigation, and also of $0.929 R_{\odot}$ by Sadik (1979) does not support the subgaint classification criterion at all. Hence, we conclude that the system UV Psc should consist of Main Sequence stars of spectral types G4-6V and $\mathrm{K0}-2 \mathrm{~V}$ in order to have an agreement with the nature of the eclipses, colours and radii of the components.

\section{Evolution}

By use of the derived masses, radii, temperatures, and luminosities of the components of UV Psc, their position on the HR-diagram is shown in Figures $7 \mathrm{a}$ and $7 \mathrm{~b}$. These figures clearly show that both the components lie very close to the Main Sequence. Hence, we can conclude that the components of UV Psc belong to the Main Sequence. This conclusion agrees with that of the other short period group of RS CVn binaries like CG Cyg (Milone and Naftilan, 1979), RT And (Mancuso et al., 1979), SV Cam (Hilditch et al., 1979), ER Vul (Al-Naimiy, 1981), and WY Cnc (Awadalla and Budding, 1979), where both the components occupy the Main Sequence domain. Hence, we suggest tentatively that the short period group of RS CVn binary components belong to the Main Sequence only.

\section{Conclusions}

Like several other RS CVn-systems, UV Psc is found to show a large intrinsic variation $\left( \pm 0^{m} .05\right)$ which cannot be accounted for by the presence of the distortion wave alone. The source of the intrinsic variation is found to be the hotter star as in the case of SS Cam and AR Lac. No periodicity of the intrinsic variation could be established with the present data. Further observations are needed to solve this problem. As neither component fills its Roche lobe, we conclude that UV Psc is a detached binary, a property it shares with other members of the RS CVn group. From the present work, the spectral types of the two components are found to be: G4-6 and $\mathrm{K} 0-2$ for the hotter and cooler components, respectively. No ultraviolet excess has been detected for either of the components. The derived colours, temperatures, absolute dimensions and nature of the eclipses strongly suggest that the components of UV Psc belong to the main sequence. This system has the same evolutionary status as other short period group of RS CVn systems like CG Cyg, RT And, SV Cam, ER Vul, and WY Cnc. 

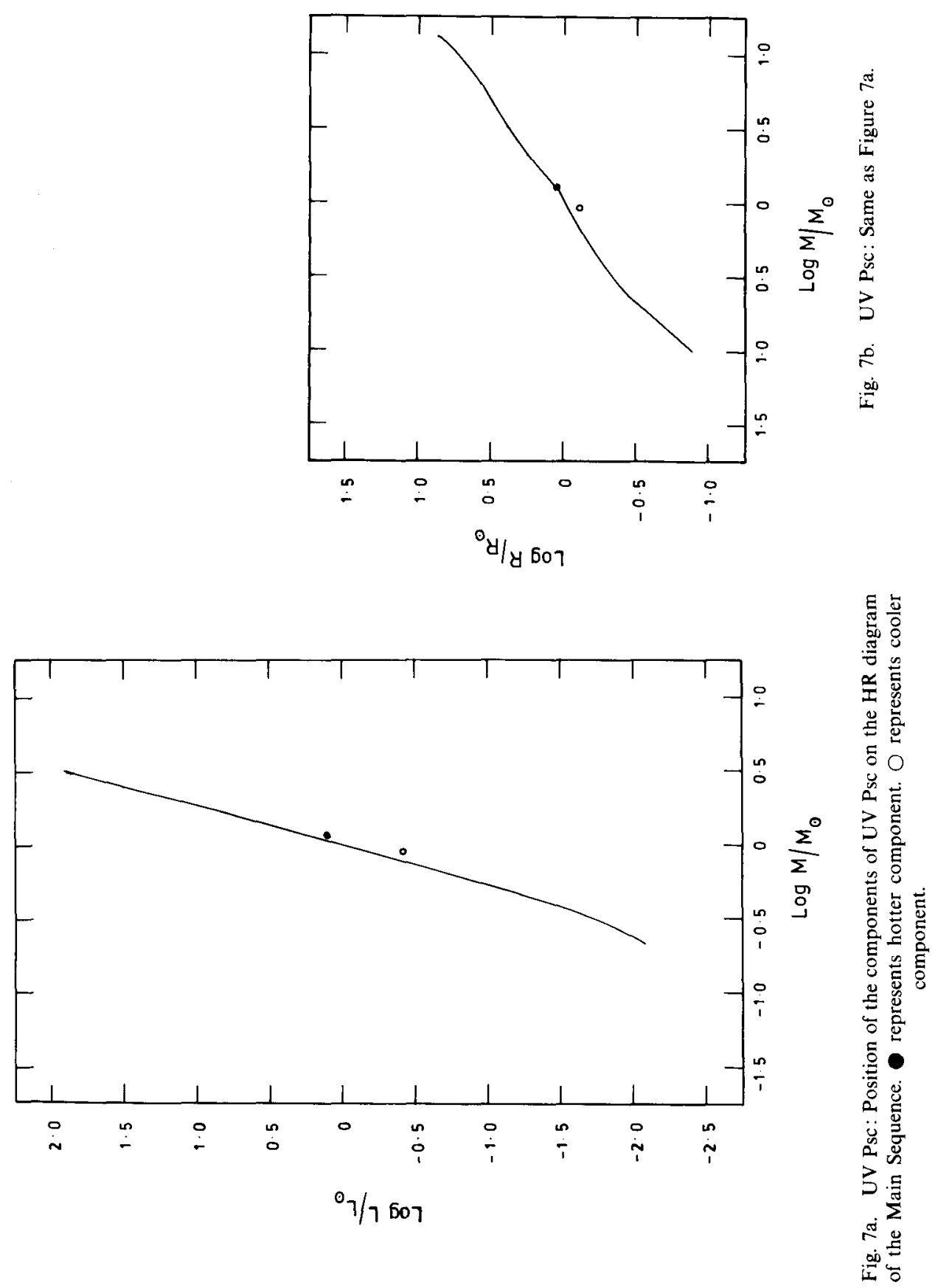
Additional spectroscopic studies of this system are needed to get a better picture regarding its spectral types, masses and evolutionary status. RI observations are needed to know more about the $\mathrm{K} 0-2$ secondary.

\section{Acknowledgements}

The authors thank Drs K. D. Abhyankar and G. C. Kilambi for useful discussions. PVR would like to thank the University Grants Commission, New Delhi, for providing financial assistance during the tenure of this work.

\section{References}

Allen, C. W.: 1976, Astrophysical Quantities, Univ. of London, The Athlone Press.

Al-Naimiy, H. M. K. : 1981, Astron. Astrophys. Suppl. 43, 85.

Arnold, C. N., Hall, D. S., Montle, R. E., and Stuhlinger, T. W.: 1979, Acta Astron. $29,243$.

Awadalla, N. S. and Budding, E.: 1979, Astrophys. Space Sci. 63, 479.

Carr, R. B. : 1969, Ph. D. Thesis, Univ. of Florida (unpublished).

Chambliss, C. R.: 1976, Publ. Astron. Soc. Pacific 88, 762.

Cester, B.: 1969, Mem. Soc. Astron. Ital. 40, 169.

Dworak, T. Z.: 1973, Inf. Bull. Var. Stars, No. 846.

Hall, D. S.: 1976, in W. S. Fitch (ed.), 'Multiple Periodic Variable Stars, Part I', IAU Colloq. 29, 287.

Hall, D. S.: 1981, in A. K. Dupree and M. Bonnet (eds.), Solar Phenomena in Stars and Stellar Systems, Reidel Publ. Co., Dordrecht, Holland, p. 431

Hilditch, R. W., Harland, D. M., and Mclean, B. J.: 1979, Monthly Notices Roy. Astron. Soc. $187,797$.

Huth, H.: 1959, Mitteilungen über veränderliche Sterne, Nr. 424.

Jakate, S., Bakos, G. A., Fernie, J. D., and Heard, J. F.: 1976, Astron. J. 81, 250.

Koch, R. H., Plavec, M., and Wood, F. B.: 1970, A Catalogue of Graded Photometric Studies of Close Binaries, Publication of the Univ, of Pennsylvania, Astronomical Series, Vol. XI.

Kopal, Z.: 1959, Close Binary Systems, Chapman and Hall, London and Wiley, New York.

Mancuso, S., Milano, L., and Russo, G.: 1979, Astron. Astrophys. Suppl 3, 415

Mardirossian, F., Mezzetti, M., Cester, B., and Giuricin, G.: 1980, Astron. Astrophys. Suppl. 39, 73.

Milone, E. F. and Naftilan, S. A.: 1979, in M. J. Plavec, D. M. Popper, and R. K. Ulrich (eds.), 'Close Binary Stars: Observations and Interpretation', IAU Symp. 88, 419.

Oliver, J. P.: Ph. D. Thesis, Univ. of California, Los Angeles (unpublished).

Plavec, M. and Kratochvíl, P.: 1964, Bull. Astron. Inst. Czech. 15, 165.

Popper, D. M.: 1956, Astrophys. J. 124, 196.

Popper, D. M.: 1961, Astrophys. J. 133, 148.

Popper, D. M.: 1976, Inf. Bull. Var. Stars, No. 1083.

Rodono, M.: 1981, in E. B. Carling and Z. Kopal (eds.), Photometric and Spectroscopic Binary Systems, Reidel Publ. Co., Dordrecht, Holland, p. 285.

Russell, H. N. and Merrill, J. E.: 1952, Contr. Princeton Univ. Obs., No. 26.

Sadik, A. R.: 1979, Astrophys. Space Sci. 63, 319.

Strohmeier, W. and Knigge, R.: 1960, Veröffentlichungen der Remeis Sternwarte ZU, Bamberg, VCS.

Tsesevich, V. P.: 1940, Bull. Astron. Inst. USSR Acad. Sci, No. 45.

Vivekananda Rao, P. and Sarma, M. B. K.: 1981a, Acta Astron. 31, 107.

Vivekananda Rao, P. and Sarma, M. B. K.: 1981b, Contributions form Nizamiah and Japal-Rangapur Observatories, No. 14.

Vivekananda Rao, P. and Sarma, M. B. K.: 1981c, in E. B. Carling and Z. Kopal (eds.), Photometric and Spectroscopic Binary Systems, D. Reidel Publ. Co., Dordrecht, Holland, p. 305.

Vivekananda Rao, P. and Sarma, M. B. K.: 1983a, Bull. Astr. Soc. India 11, 75.

Vivekananda Rao, P. and Sarma, M. B. K.: 1983b, J. Astrophys. Astron. (in press).

Wood, D. B.: 1971, Publ. Astron. Soc. Pacific 83, 286. 